\title{
Simulating Surfactant-Iron Oxide Interfaces: From Density Functional Theory to Molecular Dynamics
}

\author{
Carlos Ayestaran Latorre, James Ewen, Chiara Gattinoni, Daniele Dini
}

Submitted date: 26/06/2019 - Posted date: 26/06/2019

Licence: CC BY-NC-ND 4.0

Citation information: Latorre, Carlos Ayestaran; Ewen, James; Gattinoni, Chiara; Dini, Daniele (2019): Simulating Surfactant-Iron Oxide Interfaces: From Density Functional Theory to Molecular Dynamics. ChemRxiv. Preprint.

Understanding the behaviour of surfactant molecules on iron oxide surfaces is important for many industrial applications. Molecular dynamics (MD) simulations of such systems have been limited by the absence of a force-feild (FF) which accurately describes the molecule-surface interactions. In this study, interaction energies from density functional theory (DFT) $+U$ calculations with a van der Waals functional are used to parameterize a classical FF for MD simulations of amide surfactants on iron oxide surfaces. The Original FF, which was derived using mixing rules and surface Lennard-Jones (LJ) parameters developed for nonpolar molecules, were shown to signi cantly underestimate the adsorption energy and overestimate the equilibrium adsorption distance compared to DFT. Conversely, the Optimized FF showed excellent agreement with the interaction energies obtained from DFT calculations for a wide range of surface coverages and molecular conformations near to and adsorbed on a-Fe2O3(0001). This was facilitated through the use of a Morse potential for strong chemisorption interactions, modi fied LJ parameters for weaker physisorption interactions, and adjusted partial charges for the electrostatic interactions. The Original FF and Optimized FF were compared in classical nonequilibrium molecular dynamics (NEMD) simulations of amide molecules con fined between iron oxide surfaces. When the Optimized FF was employed, the amide molecules were pulled closer to the surface and the orientation of the headgroups was more similar to that observed in the DFT calculations compared to the Original FF. The Optimized FF proposed here facilitates classical MD simulations of amide-iron oxide interfaces in which the interactions are representative of accurate DFT calculations.

File list (2)

JPCB-MS.pdf (17.22 MiB)

view on ChemRxiv • download file 


\title{
Simulating Surfactant-Iron Oxide Interfaces:
}

\section{From Density Functional Theory to Molecular}

\section{Dynamics}

\author{
Carlos Ayestarán Latorre, ${ }^{\dagger, \uparrow}$ James P. Ewen, ${ }^{*, \dagger}$, Chiara Gattinoni, ${ }^{\dagger, \dagger}$ and \\ Daniele Dini ${ }^{\dagger}$ \\ $\dagger$ Department of Mechanical Engineering, Imperial College London, London SW7 2AZ, UK \\ $\ddagger$ Department of Materials, ETH Zürich, Zürich 8092, Switzerland \\ IThese authors contributed equally to this work \\ E-mail: j.ewen@imperial.ac.uk
}

\begin{abstract}
Understanding the behaviour of surfactant molecules on iron oxide surfaces is important for many industrial applications. Molecular dynamics (MD) simulations of such systems have been limited by the absence of a force-field (FF) which accurately describes the molecule-surface interactions. In this study, interaction energies from density functional theory $(\mathrm{DFT})+U$ calculations with a van der Waals functional are used to parameterize a classical FF for MD simulations of amide surfactants on iron oxide surfaces. The Original FF, which was derived using mixing rules and surface Lennard-Jones (LJ) parameters developed for nonpolar molecules, were shown to significantly underestimate the adsorption energy and overestimate the equilibrium adsorption distance compared to DFT. Conversely, the Optimized FF showed excellent agreement with the interaction energies obtained from DFT calculations for a wide
\end{abstract}


range of surface coverages and molecular conformations near to and adsorbed on $\alpha$ $\mathrm{Fe}_{2} \mathrm{O}_{3}(0001)$. This was facilitated through the use of a Morse potential for strong chemisorption interactions, modified LJ parameters for weaker physisorption interactions, and adjusted partial charges for the electrostatic interactions. The Original FF and Optimized FF were compared in classical nonequilibrium molecular dynamics (NEMD) simulations of amide molecules confined between iron oxide surfaces. When the Optimized FF was employed, the amide molecules were pulled closer to the surface and the orientation of the headgroups was more similar to that observed in the DFT calculations compared to the Original FF. The Optimized FF proposed here facilitates classical MD simulations of amide-iron oxide interfaces in which the interactions are representative of accurate DFT calculations.

\section{Introduction}

An improved understanding of the behavior of surfactant molecules on iron oxide surfaces would benefit a range of applications; from stabilizing iron oxide nanoparticles for use as biomarkers and catalysts, ${ }^{1}$ to extracting iron ore for steel production, ${ }^{2}$ and to protecting steel surfaces for corrosion inhibition. ${ }^{3}$ Another pertinent application where the surfactantiron oxide interface is of particular importance are friction modifier additives to lubricate steel surfaces. ${ }^{4}$ Friction modifiers are added to lubricants to reduce friction and wear in machine components, ${ }^{4}$ increase the energy efficiency of engineering systems, and ultimately reduce fuel consumption and $\mathrm{CO}_{2}$ emissions. ${ }^{5}$

Within this class of additives, organic friction modifiers (OFMs) are notable because they are based solely on $\mathrm{C}, \mathrm{H}, \mathrm{O}$, and $\mathrm{N}$ atoms and are not environmentally harmful. OFMs are amphiphilic surfactant molecules that contain nonpolar aliphatic tailgroups attached to polar headgroups. ${ }^{4}$ They adsorb to metal, metal oxide, ceramic, or carbon-based surfaces through their polar headgroups, and can eventually form high coverage monolayers. ${ }^{6,7}$ These monolayers prevent the direct contact of opposing sliding surfaces due to van der Waals 
(vdW) forces between proximal nonpolar tailgroups. ${ }^{4}$

The majority of engine components are made from steel, which forms iron oxide surfaces when exposed to air. ${ }^{8}$ Therefore, a wide range of experiments have been used to investigate the nanoscale structure of surfactant films on iron oxide surfaces; ${ }^{4}$ from polarized neutron reflectometry $(\mathrm{PNR})^{7}$ to the quartz crystal micro-balance (QCM). ${ }^{9}$ The nature and strength of surfactant adsorption on iron oxide has also been investigated through FTIR, ${ }^{10,11} \mathrm{X}$ ray photoelectron spectroscopy (XPS) ${ }^{10,12,13}$ and polarization modulation-infrared reflection absorption spectroscopy (PM-IRRAS) ${ }^{13}$ experiments. Moreover, the friction of surfactant films on iron oxide surfaces has been studied experimentally at both the nanoscale ${ }^{14}$ and the macroscale. ${ }^{4,15}$

In addition to these experimental studies, classical molecular dynamics (MD) simulations have given unique insights into the nanoscale behaviour of OFM additive films. ${ }^{16}$ For example, Doig et al. ${ }^{17,18}$ and Ewen et al. ${ }^{19-21}$ have used MD simulations to investigate the nanoscale structure and friction behavior of a range of OFM molecules on $\alpha-\mathrm{Fe}_{2} \mathrm{O}_{3}$ surfaces. The accuracy of all MD simulations is heavily dependent on the force field (FF) used to describe the interatomic interactions. ${ }^{20,22,23}$ Significant effort has been devoted to parameterizing FFs to accurately reproduce the bulk thermodynamic properties of a wide range of organic liquids. ${ }^{24-29}$ However, relatively fewer FFs have been parameterized to accurately represent the interactions between organic molecules and solid surfaces. ${ }^{30}$ In MD simulations, the interfacial vdW and electrostatic interactions are usually represented through Lennard-Jones (LJ) ${ }^{31}$ and Coulomb potentials. For example, INTERFACE-FF ${ }^{32}$ provides LJ and partial charge parameters as a transferable extension for the non-bonded interactions between organic liquids (OPLS, ${ }^{24} \mathrm{CHARMM},{ }^{28} \mathrm{AMBER},{ }^{27}$ etc.) and several silicate, aluminate, metal, oxide, sulfate, and apatite surfaces; however, no parameters for iron oxide were included. Similarly, CLAY-FF ${ }^{33}$ includes LJ and partial charge parameters for many metal hydroxide, oxyhydroxide, and clay surfaces, including iron oxides. However, these parameters were developed primarily to model water adsorption and the suitability of 
ClayFF for interactions at the surfactant-iron oxide interface remains uncertain. Previous MD simulations of surfactant-iron oxide systems ${ }^{9,17-21}$ have thus relied upon the use of mixing rules from LJ and partial charge parameters that were developed for the adsorption of $n$-alkanes. ${ }^{34,35}$

First principles calculations, such as density functional theory (DFT), can be used to accurately determine the nature and strength of the interactions between molecules and surfaces. ${ }^{36-40}$ Optimal parameters can be developed to match interaction energies, $E_{\text {int }}$, obtained from accurate DFT calculations in so-called interface FFs. ${ }^{30}$ Interface FFs have been developed to facilitate accurate classical MD simulations of several molecules on iron oxide surfaces; for example, water ${ }^{41} n$-alkanes, ${ }^{42}$ and refrigerants. ${ }^{43}$ However, no interface FF has been developed for surfactant molecules on iron oxide surfaces.

Recent DFT calculations ${ }^{40}$ have provided crucial insights into the interactions between surfactants (carboxylic acid, amide, mono-glyceride) and $\alpha-\mathrm{Fe}_{2} \mathrm{O}_{3}(0001)$ surfaces. Specifically, all of these surfactants were found to strongly chemisorb on the iron oxide surface. The carboxylic acid and glyceride molecules readily dissociated through the formation of a surface hydroxyl group, which would complicate the development of a classical FF for MD simulations. Conversely, the amide remained intact, making it a simpler target molecule for interface FF parameterization. In this study, an amide-iron oxide interface FF will be parameterized to match $E_{\text {int }}$ values obtained from previous ${ }^{40}$ and new DFT calculations of a wide range of amide surface coverages and molecular conformations near to and adsorbed on $\alpha-\mathrm{Fe}_{2} \mathrm{O}_{3}(0001)$. Starting from an Original FF using parameters from the literature, ${ }^{34}$ important parameters will be modified, and some interactions overhauled, to maximize agreement with the accurate DFT calculations.

The interface FF is expected to be useful for classical MD simulations of a wide range of applications where amide-iron oxide interactions are important, such as; stabilizing nanoparticles, ${ }^{1}$ extracting iron ore ${ }^{2}$ corrosion inhibition, ${ }^{3}$ and lubrication. ${ }^{4}$ As a final validation, the Optimized FF will be compared to the Original FF in large-scale NEMD simulations to in- 
vestigate the structure, flow, and friction of amide films on iron oxide surfaces. ${ }^{19}$ Studying the nanoscale structure of the absorbed films shows that the orientation of the headgroup changes and the molecules are drawn closer to the surface when the Optimized FF is employed. However, for the preformed films studied here, which cannot be squeezed out of the contact, the flow and friction behaviour is only slightly affected, which validates previous work which used the Original FF. ${ }^{17-21}$

\section{Methodology}

The methodology for the DFT calculations will be discussed first, followed by details of the parameterization of the Optimized FF, and finally utilization of the Optimized FF in large-scale NEMD simulations.

\section{DFT calculations}

\section{Procedure}

The procedure for the DFT $+U$ calculations was outlined in one of our recent publications. ${ }^{40}$ Therefore, only the most important aspects of the methodology are given here, while full details can be found in Ref. ${ }^{40}$

The VASP software ${ }^{44-46}$ was used with the projector augmented wave (PAW) method ${ }^{47}$ and spin polarization. For Fe, 8 electrons were treated as valance electrons, 6 for $\mathrm{O}, 4$ for $\mathrm{C}$, 7 for $\mathrm{N}$, and 1 for $\mathrm{H}$. A non-local correlation functional was employed which includes vdW interactions; optB86b-vdW. ${ }^{48,49}$ This vdW functional has been shown to perform extremely well in a wide range of molecular adsorption studies. ${ }^{36-39}$ The plane-wave expansion was cut off at $550 \mathrm{eV}$ and a Monkhorst-Pack k-points mesh of 4 x 4 x 1 was used for the slab systems. A Hubbard $U-J=4 \mathrm{eV}$ (in the Dudarev approach) ${ }^{50}$ was added to account for electronic correlations in the Fe 3d orbitals. This $U$ correction results in a band gap of $1.98 \mathrm{eV}$ for $\alpha$ -

$\mathrm{Fe}_{2} \mathrm{O}_{3}$, which is within the experimentally observed range (1.9-2.2 eV). ${ }^{51}$ Periodic boundary 
conditions are employed in the $x$ - and $y$-directions ${ }^{52}$ while 15 Aof vacuum was added in the $z$-direction to prevent interactions between neighboring cells. A dipole correction was also used in the $z$-direction. ${ }^{52}$

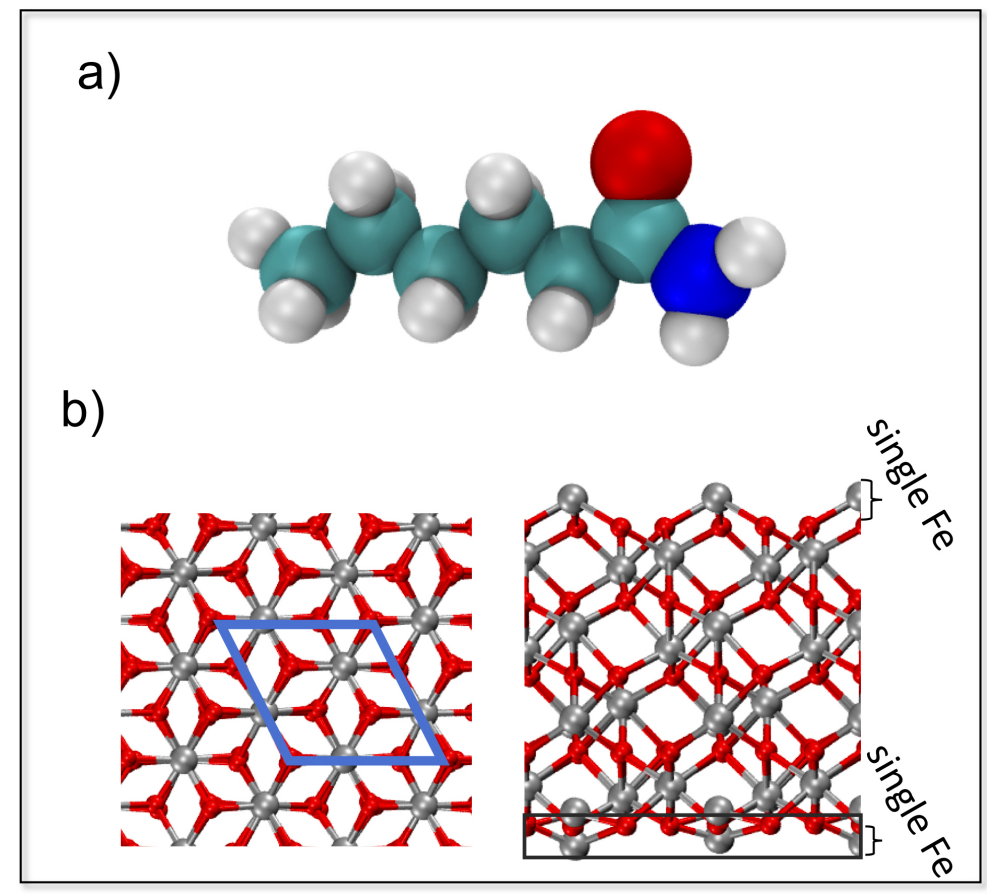

Figure 1: a) The surfactant molecule considered for the parameterization, hexanamide (HAm). b) Top (left) and side (right) view of the single Fe-terminated slab to represent the substrate for the adsorption calculations. $\mathrm{O}$ is shown in red and $\mathrm{Fe}$ in gray. In the left panel the blue box indicates the unit cell. The black box in the right panel shows the layers which are kept fixed. Visualized with VMD. ${ }^{53}$

A single Fe-terminated $\alpha-\mathrm{Fe}_{2} \mathrm{O}_{3}(0001)$ slab (shown in Fig. 1) was selected as a representative model for the surface of steel. This has been shown by numerous theoretical and experimental studies to be the most stable termination of $\alpha-\mathrm{Fe}_{2} \mathrm{O}_{3}$ at room temperature. ${ }^{54}$ The $\alpha-\mathrm{Fe}_{2} \mathrm{O}_{3}(0001)$ surface ${ }^{42}$ as well as the $\alpha-\mathrm{Fe}_{2} \mathrm{O}_{3}(00 \overline{1} 2)$ surface $^{43}$ have been used in recent FF parameterization studies. In tribology experiments, it is possible that the outer layer of $\alpha-\mathrm{Fe}_{2} \mathrm{O}_{3}(0001)$ will become hydroxylated ${ }^{55}$ due to water contamination of the lubricant. ${ }^{56}$ However, XPS experiments have suggested that surface hydroxyls can be displaced by long-chain carboxylic acids and amines on iron oxide surfaces. ${ }^{57}$ Indeed, FTIR and XPS experiments of carboxylic acid OFMs indicated that a mixture of direct carboxylate-Fe surf $_{\text {s }}$ 
bonding and carboxylate-hydroxyl $l_{\text {surf }} \mathrm{H}$-bonding occurs at the $\alpha-\mathrm{Fe}_{2} \mathrm{O}_{3}$-water interface. ${ }^{11}$ Moreover, XPS experiments of carboxylic acid ${ }^{10,13}$ and amine ${ }^{12}$ OFMs at the $\alpha$ - $\mathrm{Fe}_{2} \mathrm{O}_{3}$-oil interface showed that carboxylate- $\mathrm{Fe}_{\text {surf }}$ and dative $\mathrm{N}_{\text {amine }}-\mathrm{Fe}_{\text {surf }}$ bonding are the dominant interactions in these cases. These observations suggests that direct OFM-Fe bonding (via inner sphere complexes ${ }^{58}$ ) predominate for OFM adsorption at the $\alpha$ - $\mathrm{Fe}_{2} \mathrm{O}_{3}$-oil interface. Previous DFT calculations on $\alpha-\mathrm{Fe}_{2} \mathrm{O}_{3}(0001)$ surfaces have shown a similar adsorption energy for amide OFMs and carboxylic acid OFMs, ${ }^{40}$ so direct OFM-Fe ${ }_{\text {surf }}$ bonding can also be reasonably expected. Thus, although the steel used experimentally will present a range of surfaces terminations, ${ }^{8}$ the selected system should be sufficient to capture the key interactions governing amide adsorption at oil-steel interfaces.

Amide headgroups were chosen since they are commercially relevant and do not readily dissociate on $\alpha-\mathrm{Fe}_{2} \mathrm{O}_{3}(0001)$ surfaces. ${ }^{40}$ The tailgroups in most commercial OFMs are aliphatic chains containing 12-20 carbon atoms, mainly because they are readily available from natural fats, are soluble in most base oils, and significantly reduce friction. ${ }^{4}$ Using molecules of this size would be prohibitively expensive from a computational perspective given the large number of DFT calculations required during this study. Since the focus here is the interaction of the headgroups with the surface, the saturated tailgroups are the minimum length to ensure that the geometry and partial charges within the headgroups are representative of molecules with longer tails. Tests in our previous DFT study ${ }^{40}$ indicated that $\mathrm{C}_{6}$ groups are sufficient to achieve this, so the parameterization was performed for hexanamide (HAm), as shown in Fig. 1.

Two surface coverages of HAm molecules were considered, which can be quantified by the number of molecules per area of surface, $\Gamma$. Specifically, a low coverage (LC) of $\Gamma \sim 1 \mathrm{~nm}^{-2}$ and a high coverage (HC) of $\Gamma \sim 4 \mathrm{~nm}^{-2}$. Our previous DFT study showed that at HC, when the molecules in the monolayer are closely packed, they tend to adsorb almost vertically due vdW stabilization between neighboring tailgroups. ${ }^{40}$ Conversely, molecules prefer to adsorb flat with the tailgroups almost parallel to the surface at LC. ${ }^{40}$ An accurate representation of 
vdW forces is crucial to reproduce such behavior and tests in our previous study ${ }^{40}$ showed that the vdW interactions between two pentane molecules (models for the tailgroups) with the optB86b-vdW functional ${ }^{48,49}$ were within $3 \%$ of the MP2-calculated values. ${ }^{59}$

In total, 29 different DFT configurations were employed in the training dataset for the parameterization. The most stable relaxed structures for HAm at LC (flat), LC (vertical), and $\mathrm{HC}$ were taken from Ref. ${ }^{40}$ These configurations and the corresponding values of the adsorption energy, $E_{a d s}$, from DFT are shown in Fig. 2.
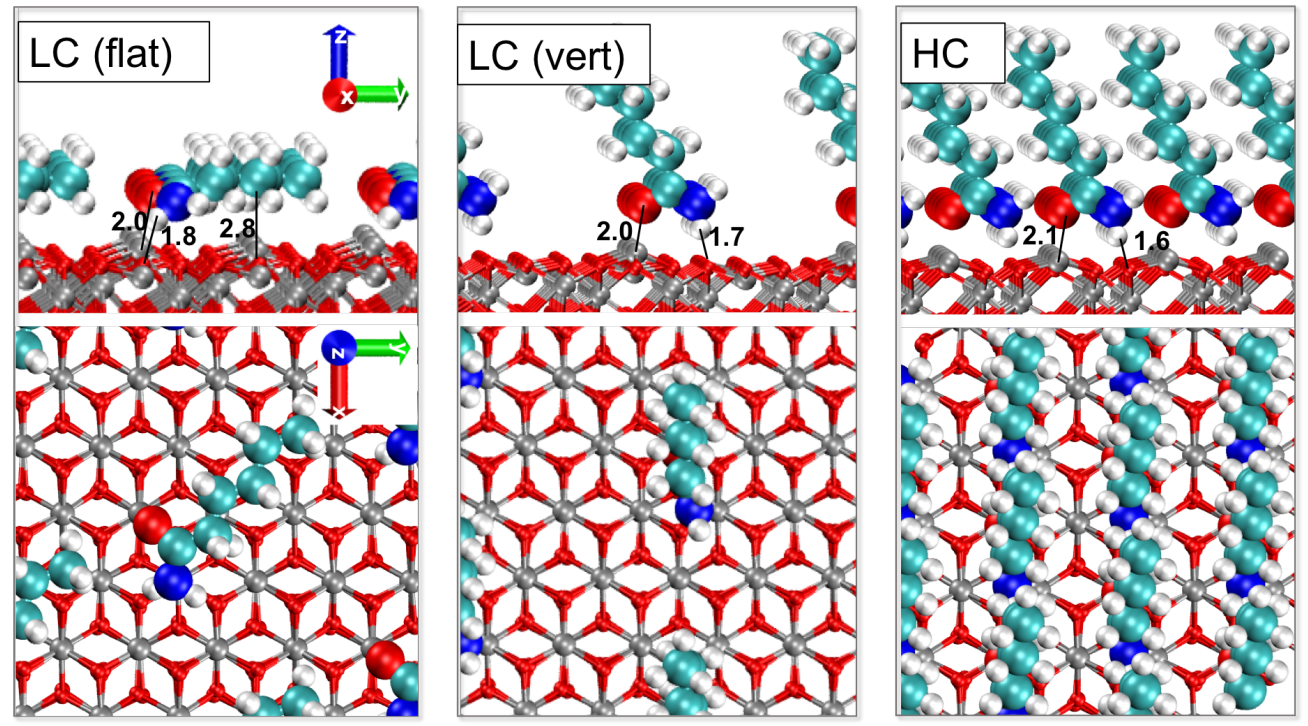

Figure 2: Side and top view of the most stable adsorption structures from Ref. ${ }^{40}$ Adsorption energy, $E_{a d s}=-60.4 \mathrm{kcal} \mathrm{mol}^{-1}$ for LC (flat), $-48.9 \mathrm{kcal} \mathrm{mol}^{-1}$ for LC (vertical), and -51.7 kcal $\mathrm{mol}^{-1}$ for HC. Marked distances between atom pairs are in $\AA$. Visualized with VMD. ${ }^{53}$

Relaxed adsorption configurations, which showed slightly weaker adsorption compared to those included in Ref., ${ }^{40}$ as shown in the SI (Fig. S1), were also included in the training dataset to prevent overfitting. ${ }^{60,61}$ These relaxed adsorption structures were further supplemented by systems in which the molecules were translated and/or rotated to ensure extensive sampling of the conformational phase space. ${ }^{30}$ Specifically, single-point DFT calculations were performed with the HAm molecules translated in the direction perpendicular to the surface (z-scan) by $1.0 \AA$ to $3.6 \AA .^{41,62-64}$ These calculations were performed on both the LC (flat) and LC (vertical) systems, to ensure that the $E_{\text {int }}$-distance profiles are accurately 
described by the Optimized FF for both cases. Single-point DFT calculations were also performed on tilted conformations as intermediates between the flat and vertical cases. ${ }^{62}$

Since $n$-alkanes are also frequently included in large-scale NEMD simulations of amideiron oxide systems, ${ }^{19} E_{a d s}$ was also calculated for $n$-hexane on $\alpha-\mathrm{Fe}_{2} \mathrm{O}_{3}(0001)$. In the most stable $n$-hexane adsorption structure obtained by structural relaxation, $E_{a d s}=-28.4$ kcal $\mathrm{mol}^{-1}$, as shown in the SI (Fig. S2). This represents relatively stronger adsorption than observed in previous DFT calculations of $n$-butane on $\alpha-\mathrm{Fe}_{2} \mathrm{O}_{3}(0001)$ which used vdW correction methods ${ }^{42}$ rather than the vdW functional employed here. ${ }^{48,49}$ The $n$-hexane molecule in the relaxed configuration was also translated in the $z$-direction to obtain a $z$-scan of $E_{\text {int }}$ for this system. Since the focus of this interface FF is the behaviour of surfactants on iron oxide, the $n$-hexane results were used to ensure comparable performance to other FFs, ${ }^{33-35}$ rather than forming part of the parameterization; as shown in the SI.

\section{Interaction energy}

The molecule-surface $E_{\text {int }}$ was calculated by: ${ }^{40}$

$$
E_{\text {int }}=\left(E_{\text {sys }}-E_{\text {surf }}-N_{\text {mol }} \times E_{\text {mol }}\right) / N_{\text {mol }}
$$

where $E_{\text {sys }}$ is the total energy of the adsorbed system, $E_{\text {surf }}$ is the total energy of the $\alpha$ $\mathrm{Fe}_{2} \mathrm{O}_{3}(0001)$ slab, $E_{m o l}$ is the total energy of the gas-phase molecule and $N_{m o l}$ is the number of HAm molecules in each cell. A negative value of $E_{\text {int }}$, therefore, indicates an energetically favorable interaction with respect to the isolated molecules and surface.

\section{Interface force-field parameterization}

\section{Original force-field}

The use of accurate all-atom FFs is critical to accurately reproduce the viscosity of bulk organic liquids ${ }^{20,22}$ as well as the structure and friction of adsorbed monolayers. ${ }^{20,65}$ Here, 
we use the optimized potentials for liquid simulations all-atom (OPLS-AA) FF, ${ }^{24,25}$ which includes parameters for the $\mathrm{N}, \mathrm{O}, \mathrm{C}$, and $\mathrm{H}$ atoms in the amide headgroups. ${ }^{19}$ For $\mathrm{C}$ and $\mathrm{H}$ atoms in the tailgroups and $n$-hexadecane molecules, updated parameters from L-OPLS-AA $\mathrm{FF}^{26}$ are employed. L-OPLS-AA is a refinement of OPLS-AA specifically for long-chain alkanes, which significantly improves the agreement with experimental density and viscosity values for such molecules. ${ }^{20}$ The bonded and non-bonded parameters for the interactions between the amide and $n$-hexadecane molecules are not changed during the parameterization. Thus, only non-bonded interfacial interactions between atoms in the amide molecules with the iron oxide surface atoms are directly parameterized. ${ }^{41-43}$ In most FFs, such interactions are described using the $12-6 \mathrm{LJ}^{31}$ and Coulombic pair potentials: ${ }^{30,32}$

$$
E_{\text {non-bonded }}=E_{L J}+E_{C}=\sum_{i, j>i}\left[4 \epsilon_{i j}\left[\left(\frac{\sigma_{i j}}{r_{i j}}\right)^{12}-\left(\frac{\sigma_{i j}}{r_{i j}}\right)^{6}\right]+\frac{C q_{i} q_{j}}{r_{i j}}\right]
$$

where $C$ is an energy-conversion constant, $q_{i}$ and $q_{j}$ are the partial charges of atoms $i$ and $j$, $r_{i j}$ is the distance between atoms $i$ and $j, \epsilon_{i j}$ is the depth of the LJ potential well between atoms $i$ and $j, \sigma_{i j}$ is the distance at which the inter-particle LJ potential is zero. Each atom type is assigned unique $q, \epsilon$, and $\sigma$ parameters.

As a starting point (Original FF), LJ and partial charge parameters for the $\mathrm{Fe}_{\text {surf }}$ and $\mathrm{O}_{\text {surf }}$ atoms $\left(\epsilon_{\mathrm{Fe}}=2.5 \times \epsilon_{O}\right)$ were taken from the $\mathrm{FF}$ due to Berro et al. ${ }^{34} \mathrm{LJ}$ interactions between dissimilar atoms are obtained using geometric mean mixing rules; $\sigma_{i j}=\sqrt{\sigma_{i} \sigma_{j}}, \epsilon_{i j}=$ $\sqrt{\epsilon_{i} \epsilon_{j}}$, as specified for the OPLS-AA FF. ${ }^{24}$ The choice of this FF over ClayFF ${ }^{33}$ and the FF due to Savio et al. ${ }^{35}$ is rationalized in the SI (Fig. S3). The LJ and partial charge parameters in the Original FF were developed to model the interactions between $n$-hexadecane molecules and $\mathrm{Fe}_{2} \mathrm{O}_{3}$ surfaces. ${ }^{34}$ They have also been applied in several large-scale MD simulations of surfactant-iron oxide systems. ${ }^{9,17-21}$ 


\section{Interface force-field optimization procedure}

The atomic coordinates from the DFT calculations were transferred into LAMMPS ${ }^{66}$ where the energy calculations for the Original FF and Optimized FF were performed. This was facilitated using the Materials and Processes Simulations (MAPS) platform from Scienomics SARL. As for the DFT calculations, periodic boundary conditions were applied in $x$ and $y$ directions, with 15 Åf vacuum added in the $z$ direction. The DFT systems were replicated three times in the $x$ and $y$ directions to prevent the molecules from interacting with their own periodic images. ${ }^{43}$ A cutoff of $12 \AA$ was used for the LJ interactions. A slab implementation of the particle-particle particle-mesh algorithm with a relative force accuracy of $10^{-5}$ was used for the electrostatic interactions. ${ }^{67}$ Bonded and non-bonded interactions between the HAm molecules were described by the L-OPLS-AA FF. ${ }^{24-26}$

The Optimized FF parameters were obtained using a particle swarm algorithm. ${ }^{68}$ The use of a minimization algorithm provides a more systematic and efficient method compared to the trial-and-error approaches commonly used for interface FF parameterization. ${ }^{41,42}$ QuasiNewtonian algorithms, which have been used in other interface FF parameterization studies $^{43}$ and have been implemented in some parameterization software packages ${ }^{61,69}$ were also tested. However, such methods require accurate initial guesses since they are more suited to finding local minima. Conversely, particle swarm optimization can locate global minima; ${ }^{68}$ a full description of this algorithm is given in the SI. The optimization was performed using relative deviations in $E_{\text {int }}$ to the DFT values, as opposed to absolute deviations to ensure that geometries with lower $E_{i n t}$ (and the lower associated absolute errors) were given equal weight. ${ }^{30}$ Force-matching ${ }^{70,71}$ was also included in the optimization for the relaxed DFT configurations, as described in the SI.

Initially, standard 12-6 LJ and partial charge parameters were optimized, but this approach proved insufficient to represent the strong interactions observed in the DFT calculations. An extensive analysis of different potential combinations is discussed in the SI. Ultimately, a combination of 12-6 LJ, Coulombic, and the Morse potential for the chemiso- 
prtion interactions provided an excellent match to the DFT-calculated $E_{\text {int }}$ values for the wide range of configurations considered.

\section{Morse interactions}

The DFT calculations in Ref. ${ }^{40}$ showed that for HAm molecules on $\alpha-\mathrm{Fe}_{2} \mathrm{O}_{3}(0001)$ surfaces, $E_{a d s} \approx-50 \mathrm{kcal} \mathrm{mol}^{-1}$. Strong interactions between the oxygen atoms $\left(\mathrm{O}_{\text {amide }}\right)$ and nitrogen atoms $\left(\mathrm{N}_{\text {amide }}\right)$ in the amide headgroups with the surface iron atoms $\left(\mathrm{Fe}_{\text {surf }}\right)$ as well as between the headgroup hydrogen atoms $\left(\mathrm{H}_{\text {amide }}\right)$ and the surface oxygen atoms $\left(\mathrm{O}_{\text {surf }}\right)$ were observed. Fig. 3 shows that the $\mathrm{O}_{\text {amide }}-\mathrm{Fe}_{\text {surf }}, \mathrm{N}_{\text {amide }}-\mathrm{Fe}_{\text {surf }}$, and $\mathrm{H}_{\text {amide }}-\mathrm{O}_{\text {surf }}$ interactions all involve charge transfer between the headgroup and the surface atoms, which is indicative of chemisorption. ${ }^{40}$

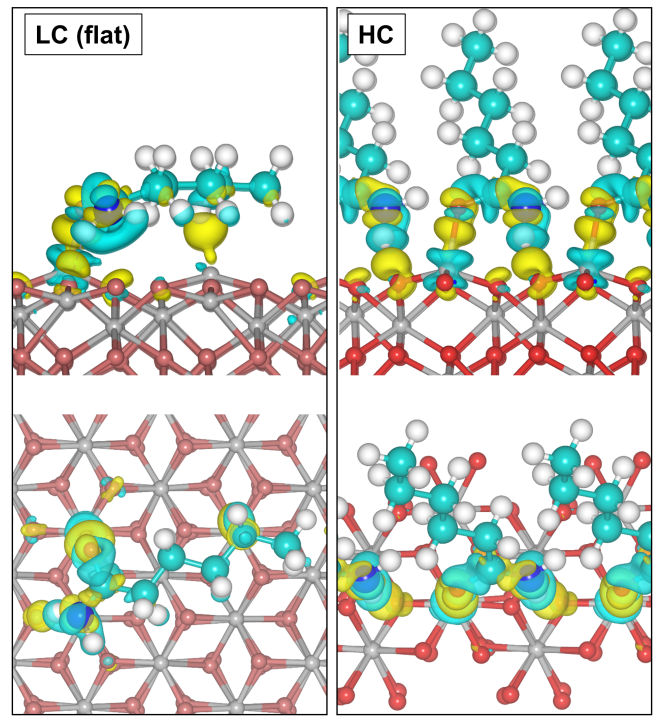

Figure 3: Side and top view of the electron density difference plots for the most stable low coverage (LC) and high coverage (HC) structures of HAm. Green represents regions of charge density depletion, while yellow represents regions of accumulation. The isosurface level is $0.003 \mathrm{e} \mathrm{a}_{0}^{-3}$ (where $\mathrm{a}_{0}$ is the Bohr radius).

The 12-6 LJ potential used in the majority of classical FFs is better suited to reproducing weaker physisorption interactions. ${ }^{62,63}$ Indeed, tests in which the surface LJ parameters and partial charges were modified from the Original FF showed only marginal improvement, as 
shown in the SI (Fig. S4-5). Therefore, to significantly improve the agreement between the Optimized FF and the DFT calculations, it was necessary to change the functional form of the $\mathrm{O}_{\text {amide }}-\mathrm{Fe}_{\text {surf }}, \mathrm{N}_{\text {amide }}-\mathrm{Fe}_{\text {surf }}$, and $\mathrm{H}_{\text {amide }}-\mathrm{O}_{\text {surf }}$ interactions. Much better agreement between the $E_{a d s}$ values from DFT calculations and the Optimized FF was obtained when the Morse potential was fit for these interactions:

$$
E_{\text {Morse }_{i j}}=D_{i j}\left[e^{-2 \alpha_{i j}\left(r_{i j}-r_{i j}^{0}\right)}-2 e^{-\alpha_{i j}\left(r_{i j}-r_{i j}^{0}\right)}\right]
$$

where $D_{i j}$ is the well depth, $r_{i j}^{0}$ is the equilibrium distance and $\alpha_{i j}$ determines the width of the potential. ${ }^{72}$ The three tunable parameters allows the Morse potential to have both a deep potential well to reproduce strong short-range binding, but also decay in a manner consistent with the DFT calculations. This is not possible with LJ potentials which have a fixed $\sim r^{-6}$ decay and only two tunable parameters. ${ }^{60}$ Moreover, the $1 / \mathrm{r}^{12}$ repulsion used in LJ is used primarily for numerical convenience, while the exponential repulsion in the Morse potential has more physical motivation. ${ }^{70}$ Indeed, the Morse potential has been shown to more accurately reproduce $E_{a d s}$ from DFT compared to LJ potentials for a wide range of molecules on solid surfaces, for example; $\alpha$-olefins (ethene to 1-decene) on $\mathrm{Al}(001)$ surfaces, ${ }^{70}$ 1,4-bis(cyanophenyl)22,5-bis(decyloxy)-benzene on $\mathrm{KCl}(100),{ }^{60}$ gases $\left(\mathrm{H}_{2}, \mathrm{O}_{2}, \mathrm{~N}_{2}, \mathrm{CO}, \mathrm{H}_{2} \mathrm{O}\right.$, $\left.\mathrm{H}_{2} \mathrm{~S}\right)$ on alkali metal-doped carbon nanotubes, ${ }^{63}$ and water on $\mathrm{Au}(111) .{ }^{64}$ Of particular relevance to this study, Morse interactions have also been used to accurately represent the S-Au chemisorption interactions of thiol molecules on Au surfaces. ${ }^{73,74}$

\section{Optimized FF parameters}

In the Optimized FF, nine parameters were fitted to Morse potentials to represent the strong $\mathrm{O}_{\text {amide }}-\mathrm{Fe}_{\text {surf }}, \mathrm{N}_{\text {amide }}-\mathrm{Fe}_{\text {surf }}$, and $\mathrm{H}_{\text {amide }}-\mathrm{O}_{\text {surf }}$ interactions. Since the interactions between the remaining atom type pairs in the surface and HAm were of a physical rather than

chemical nature, they were simultaneously fitted with a 12-6 LJ potential. It was sufficient to only change the surface LJ parameters and use geometric mean mixing rules for these 
weaker interactions rather than fitting each atom pair individually, ${ }^{43}$ so this only added four parameters to the fitting. Note that the LJ interactions are ignored for the $\mathrm{O}_{\text {amide }}-\mathrm{Fe}_{\text {surf }}$, $\mathrm{N}_{\text {amide- }}-\mathrm{Fe}_{\text {surf }}$, and $\mathrm{H}_{\text {amide }}-\mathrm{O}_{\text {surf }}$ interactions in the Optimized FF. The partial charges of the $\mathrm{Fe}_{\text {surf }}$ and $\mathrm{O}_{\text {surf }}$ atoms were also adjusted to maximize agreement with the $E_{\text {ads }}$ values from the DFT calculations. A charge neutrality constraint was imposed on the surface (Fe:O charge ratio $=3: 2)$, so only one additional parameter was required to determine the $\mathrm{Fe}_{\text {surf }}$ and $\mathrm{O}_{\text {surf }}$ partial charges. In total, 14 parameters were included in the fitting procedure.

The final parameters for the Optimized FF are presented in Table 1. The parameters are in general agreement with those developed previously for other molecular adsorption systems. The Morse parameters for the $\mathrm{O}_{\text {amide }}-\mathrm{Fe}_{\text {surf }}$ and $\mathrm{N}_{\text {amide }}-\mathrm{Fe}_{\text {surf }}$ are broadly similar to those developed for S-Au interactions of thiol molecules on Au surfaces. ${ }^{73,74}$ Experimental values of $\mathrm{E}_{\text {int }}$ are not available for amides; however, XPS experiments of amines showed that dative $\mathrm{N}_{\text {amine }}-\mathrm{Fe}_{\text {surf }}$ bonding dominates the interactions, ${ }^{12}$ while carboxylate-Fe $\mathrm{surf}_{\text {s }}$ bonding did so for carboxylic acids. ${ }^{10,13} \mathrm{MP} 2$ level calculations have shown that $\mathrm{E}_{\text {int }} \approx 5 \mathrm{kcal} \mathrm{mol}^{-1}$ for amide-water $\mathrm{H}$-bonds (when water is the $\mathrm{H}$-bond acceptor), ${ }^{75}$ which is similar to the $\mathrm{H}_{\text {amide }}-\mathrm{O}_{\text {surf }}$ Morse parameters used here. The LJ and partial charge parameters are only slightly changed from those used in the Original FF ${ }^{34}$ (see Table S1).

Table 1: Final LJ, Morse and partial charge parameters for Optimized FF

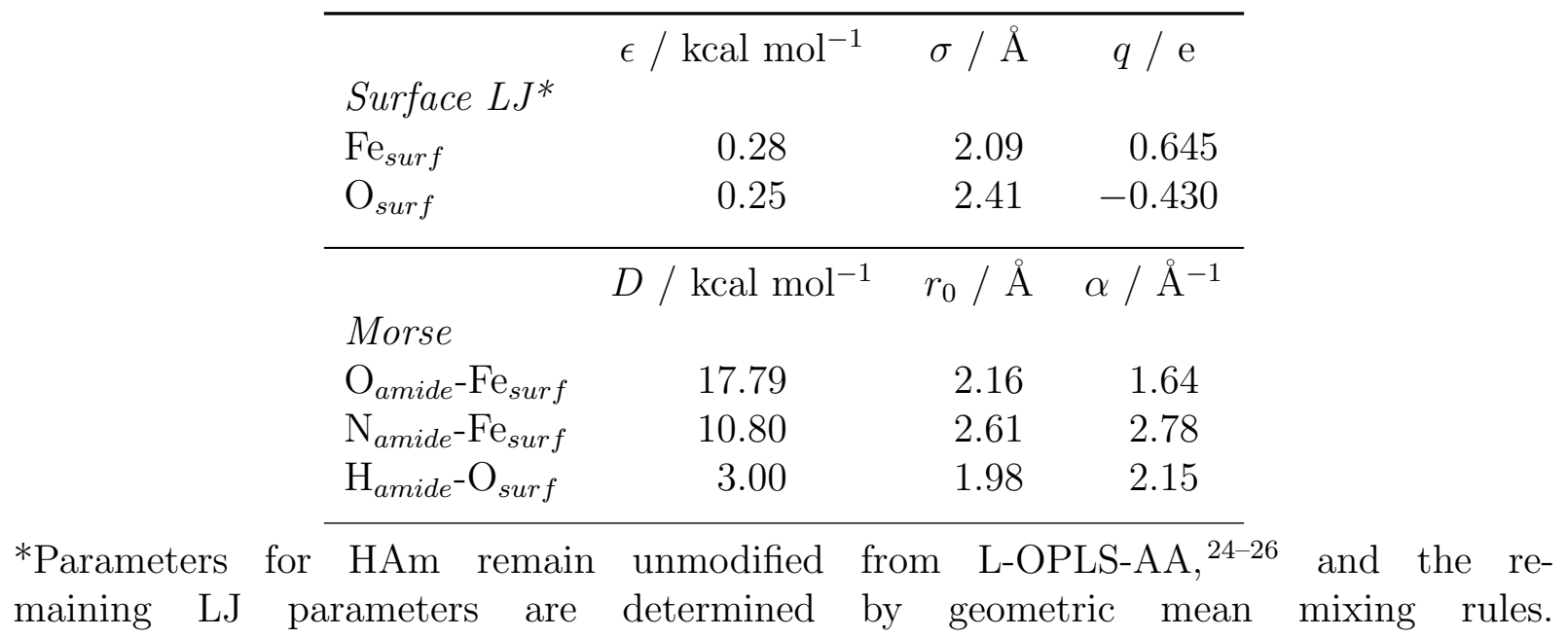




\section{Large-scale NEMD simulations}

\section{Simulation set-up}

As a final validation, the Optimized FF was tested in large-scale confined NEMD simulations of amide films adsorbed on $\alpha-\mathrm{Fe}_{2} \mathrm{O}_{3}$ surfaces, separated by a thin layer of $n$-hexadecane. In previous simulations of similar systems, ${ }^{17-21}$ the molecule-surface interactions were represented by LJ and Coulomb potentials, with the $\alpha-\mathrm{Fe}_{2} \mathrm{O}_{3}$ surface parameters taken from Berro et al. ${ }^{34}$ In this section, this Original FF (Table S1) will be compared to the Optimized FF (Table 1) in large-scale NEMD simulations. The L-OPLS-AA ${ }^{24-26} \mathrm{FF}$ was used to represent interactions between the amide and $n$-hexadecane molecules.

The simulation procedure is similar to that described in one of our previous studies. ${ }^{19}$ Therefore only the most important details are included here, while the full methodology can be found in Ref. ${ }^{19}$ The systems consist of a thin layer of $n$-hexadecane lubricant confined between amide films adsorbed on two $\alpha-\mathrm{Fe}_{2} \mathrm{O}_{3}(0001)$ slabs with $x, y, z$ dimensions of 55, 55, 12 $\AA$. Periodic boundary conditions were applied in the $x$ and $y$ directions, so the amide or $n$ hexadecane molecules could not be squeezed out of the contact. ${ }^{76}$

The NEMD simulations were performed using LAMMPS, ${ }^{66}$ and the equations of motion were integrated with the velocity Verlet algorithm with a time step of $1 \mathrm{fs}$. Fast-moving bonds involving hydrogen atoms were constrained with the SHAKE algorithm. ${ }^{77}$ The $\alpha$ $\mathrm{Fe}_{2} \mathrm{O}_{3}$ slab atoms were restrained by harmonic bonds between atoms within $3 \AA$, with a force constant of $130 \mathrm{kcal} \mathrm{mol}^{-1} \AA^{-1}{ }^{34}$

Stearamide (SAm), which has a $\mathrm{C}_{18}$ tailgroup rather than $\mathrm{C}_{6}$ tailgroup in HAm, was used since it is more representative of commercial OFMs. ${ }^{4}$ SAm molecules were placed $3 \AA$ from both of the solid surfaces prior to energy minimization. This is similar to the use of preformed Langmuir-Blodgett films in friction experiments. ${ }^{78}$ Three different surface coverages of SAm

were considered. A HC, where $\Gamma=4.32 \mathrm{~nm}^{-2}$ (close to the maximum theoretical value) ${ }^{79}$ a MC, where $\Gamma=2.88 \mathrm{~nm}^{-2}(\sim 2 / 3$ maximum $)$; and a LC, where $\Gamma=1.44 \mathrm{~nm}^{-2}(\sim 1 / 3$ 
maximum). 70 -hexadecane molecules, equivalent to two molecular layers, were randomly inserted between the SAm films. This has been shown in previous squeeze-out simulations to be representative of the amount expected between OFM films under boundary lubrication conditions at $0.5 \mathrm{GPa} .{ }^{19}$

\section{Simulation Procedure}

The large-scale NEMD simulations consisted of three phases; minimization, compression and sliding. Starting with a density similar to that of liquid $n$-hexadecane $\left(0.75 \mathrm{~g} \mathrm{~cm}^{-3}\right)$, the system was energy minimized. The system was then pressurized (0.5 GPa) and it was thermostatted $(300 \mathrm{~K})$ in the directions perpendicular to the compression and shear directions. Temperature was maintained with a Langevin thermostat with a relaxation constant of 0.1 ps. ${ }^{80}$ Pressure was applied by adding a constant force to atoms in the outermost layer of the top slab, while keeping the outermost layer of atoms in the bottom slab fixed in the $z$ direction. The systems were compressed for 500 ps, which was sufficient for the slab separation to reach a constant average value.

A velocity of $v= \pm 10 \mathrm{~m} \mathrm{~s}^{-1}$ was added to the outermost layer of atoms in each slab such that $v_{s}=20 \mathrm{~m} \mathrm{~s}^{-1}$. Four independent NEMD simulations were run for each coverage/FF combination, with the top slab sliding in the $x,-x, y$ and $-y$ directions to account for potential friction anisotropy. ${ }^{81}$ The sliding simulations were conducted for approximately 1 ns which was sufficient to reach a nonequilibrium steady state. Any heat generated during the sliding was dissipated using a thermostat acting only on the middle $10 \AA$ of the $\alpha-\mathrm{Fe}_{2} \mathrm{O}_{3}$ slabs, applied only in the $y$-direction (perpendicular to both the sliding and compression). This approach ensures that the dynamics of the confined liquids molecules is not unphysically influenced under sliding conditions. ${ }^{82}$ Using this thermostatting method, the temperature rise within the liquid region was negligible under the studied conditions. ${ }^{19}$ 


\section{Results and Discussion}

\section{Interface force-field parameterization}

To assess the performance the Optimized FF, $E_{\text {int }}$ was compared to the Original FF and accurate DFT calculations using the optB86b-vdW functional ${ }^{48,49}$ for a wide range of configurations. Fig. 4 shows $z$-scans of $E_{\text {int }}$ for LC (flat) (a) and LC (vertical) (b) HAm configurations obtained using DFT calculations, the Original FF, and the Optimized FF. Comparisons of $z$-scans of $E_{\text {int }}$ to the DFT calculations for ClayFF ${ }^{33}$ and the FF due Savio et al. ${ }^{35}$ are shown in the SI (Fig. S3). In the DFT calculations, adsorption is stronger for the LC (flat) case $\left(E_{a d s}=-60.0 \mathrm{kcal} \mathrm{mol}^{-1}\right)$ compared to LC (vertical) $\left(E_{a d s}=-48.4 \mathrm{kcal} \mathrm{mol}^{-1}\right)$ conformation owing to vdW interactions between the tailgroups and the surface atoms. ${ }^{40}$

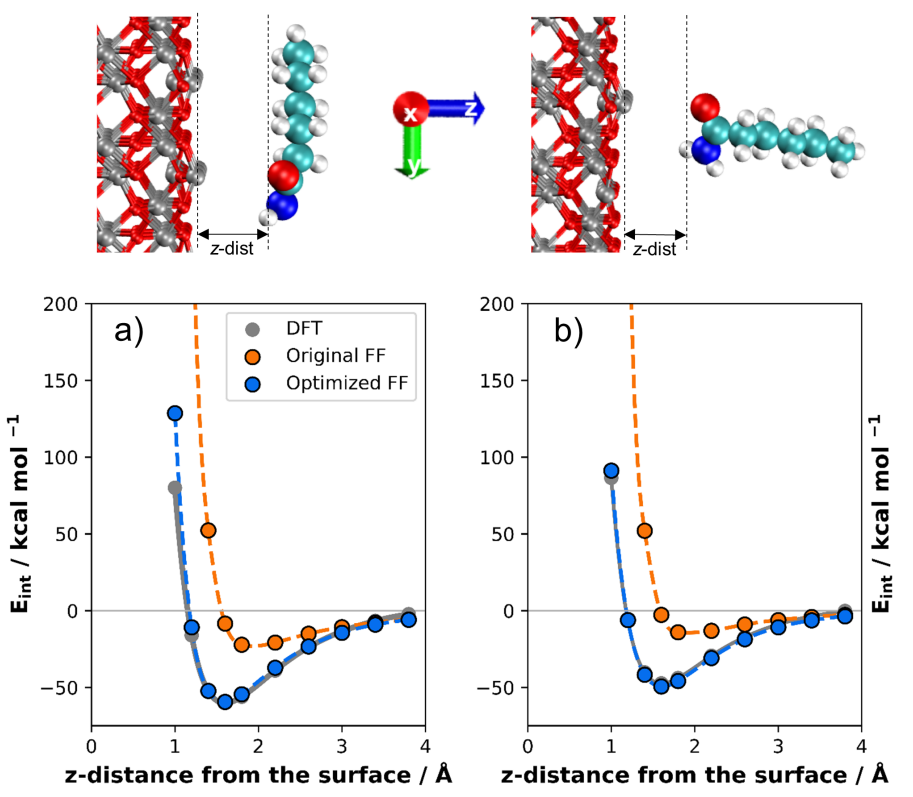

Figure 4: $z$-Scans of $E_{\text {int }}$ for the a) LC (flat) and b) LC (vertical) HAm configurations. Solid gray line is spline interpolation to DFT data, dashed orange line is Original FF, ${ }^{34}$ dashed blue line is Optimized FF.

Comparing the Original $\mathrm{FF}^{34}$ to the DFT calculations, when the HAm molecules are $\geq 2.5 \AA$ from the surface and $E_{\text {int }}$ is relatively weak, reasonable agreement is achieved for both the LC (flat) and LC (vertical) conformations. However, as the HAm molecules are 
moved closer to the surface, the agreement with DFT becomes much poorer. Firstly, the equilibrium adsorption distance, $z_{e q} \approx 1.9 \AA$ for the Original $\mathrm{FF}$, which is $\sim 0.4 \AA$ larger than the $1.5 \AA$ obtained using DFT. Moreover, $E_{a d s} \approx-20 \mathrm{kcal} \mathrm{mol}^{-1}$, which is much lower than the value obtained from DFT, $E_{a d s} \approx-50 \mathrm{kcal} \mathrm{mol}^{-1}$. As is commonly observed for LJ potentials, ${ }^{62,70,83}$ the repulsive energy at small molecule-surface separations is also considerably overestimated.

Conversely, the Optimized FF shows excellent agreement with the $z$-scan of $E_{\text {int }}$ from the DFT calculations for both the LC (flat) and LC (vertical) HAm conformations. Note that Fig. 4 also includes configurations $\leq 1.0 \AA$ from the surface which were not included in the training dataset. The repulsive energy for these configurations is overestimated as a result of the unphysically steep repulsive term in the 12-6 LJ potential. ${ }^{62,70,83}$ However, the overestimation for the Optimized FF is much less severe than for the Original FF. It is noteworthy that the very high energy configurations are unlikely to be accessed in MD simulations and therefore the accuracy of these energies is less important. ${ }^{62}$ Nevertheless, the Optimized FF will correctly predict repulsive energies in order to avoid sampling unrealistic configurations in the MD simulations. To match the DFT energies for the closest-ranged configurations, Morse parameters would need to be fit for all of the non-bonded interactions; ${ }^{83}$ however, this would complicate the optimization procedure and would risk overfitting. ${ }^{60,61}$ Further improvements could perhaps be obtained by using a more computationally expensive polarizable $\mathrm{FF}^{69}$ rather than the partial charge model employed here for the electrostatic interactions.

Although it was not used in the parameterization, it was also ensured that $z$-scan for a relaxed $n$-hexane molecule on $\alpha-\mathrm{Fe}_{2} \mathrm{O}_{3}(0001)$ was accurately reproduced with the Optimized FF. For both the Original FF and Optimized FF, $E_{a d s}$ for $n$-hexane on was underestimated compared to that obtained from the DFT calculations, as shown in the SI (Fig. S6). To improve agreement with the DFT calculations for $n$-hexane, $\epsilon_{F e}$ could be increased, similar to the approach used by Savio et a. ${ }^{35}$ However, this would negatively influence the agreement 
of the Optimized FF with the DFT results for the HAm molecules, which was the main aim of this study. Alternatively, improved agreement could be obtained by developing Morse parameters for the interaction of $\mathrm{C}$ and $\mathrm{H}$ atoms in $n$-hexane with the surface atoms, ${ }^{83}$ but this is beyond the scope of this current study.

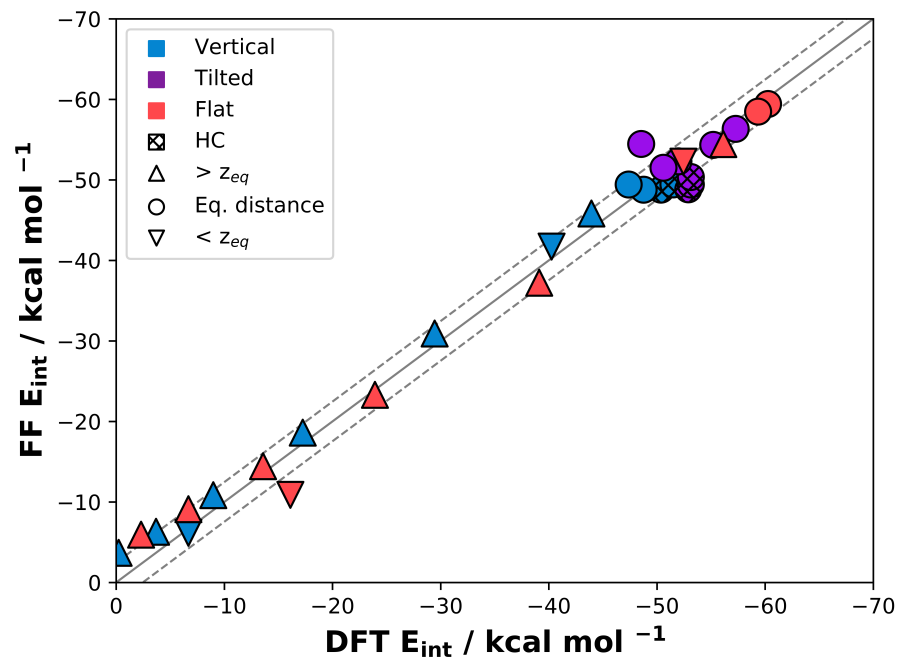

Figure 5: Comparison of the interaction energy, $E_{\text {int }}$, from the Optimized FF and DFT calculations for all of the configurations investigated. Solid line represents perfect fitting, dashed lines represent the RMS error of $2.45 \mathrm{kcal} \mathrm{mol}^{-1}$.

A comparison between the $E_{\text {int }}$ values obtained from the Optimized FF and the DFT calculations for all of the configurations included in the parameterization is shown in Fig. 5. The linear fit of these data has an $R^{2}$ value of 0.98 , representing a dramatic improvement over the Original $\mathrm{FF}^{34}$ and other alternatives shown in the SI (Fig. S3-4). Overall, the deviations between the FF and DFT energies amount to a root mean square (RMS) error of only $2.5 \mathrm{kcal} \mathrm{mol}^{-1}$, which is an order of magnitude smaller than the strength of the interactions. Thus, the performance of the Optimized FF compares favorably with interface FFs developed for other molecule-surface combinations where direct comparisons between $E_{\text {int }}$ from the FF and DFT were conducted. ${ }^{43,62,64}$ 


\section{Large-scale NEMD simulations}

In the final stage, the Optimized FF was compared to the Original $\mathrm{FF}^{34}$ in large-scale NEMD simulations of amide films adsorbed on $\alpha-\mathrm{Fe}_{2} \mathrm{O}_{3}(0001)$ surfaces at $300 \mathrm{~K}$. Fig. 6 shows number density profiles for the SAm headgroup (amide C, O, H, N) atoms which give indications regarding the orientation of the headgroups. Number density profiles shown for the top wall moving in the $+x$ direction, results for $-x,+y$, and $-y$ are shown in the SI (Fig. S7-9).
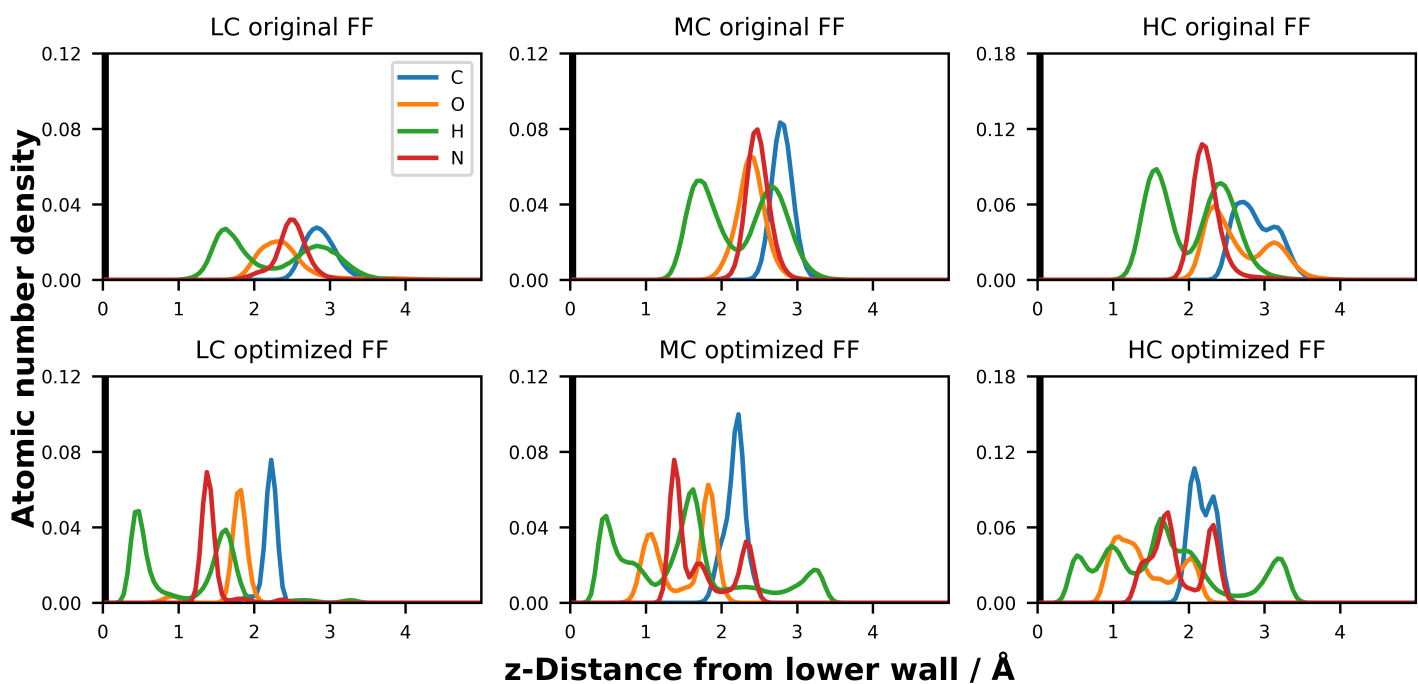

Figure 6: Number density profiles for SAm and $n$-hexadecane atoms for the Original FF (top) and optimized FF (bottom) under sliding conditions. Top wall moved in the $+x$ direction.

Generally, the atomic number density peaks in Fig. 6 become more intense as the coverage is increased from LC to $\mathrm{HC}$ for both the Original FF and the Optimized FF, which is indicative of more ordered molecules. For the Original FF, there are two $\mathrm{H}_{\text {amide }}$ peaks in Fig. 6 which have similar intensity to each other at LC, MC, and HC. The Original FF also shows single $\mathrm{O}_{\text {amide }}$ and $\mathrm{N}_{\text {amide }}$ peaks which are approximately equidistant from the surface at all coverages considered. This is unsurprising since these atoms have similar LJ parameters $\left(\sigma_{O}=2.96 \AA, \epsilon_{O}=0.21 \mathrm{kcal} \mathrm{mol}^{-1}, \sigma_{N}=3.25 \AA, \epsilon_{N}=0.17 \mathrm{kcal} \mathrm{mol}^{-1}\right)^{24}$ which govern the interaction strength and distance with the surface atoms. Previous NEMD studies with the Original $\mathrm{FF}^{19}$ have shown that the SAm tailgroups become more upright as 
the coverage is increased. However, these current results suggest that the headgroups remain in a similar conformation as the coverage is increased.

The DFT calculations suggest that the headgroup conformation is actually rather sensitive to surface coverage, as shown in Fig. 2. In the most stable $\left(\mathrm{E}_{a d s}=-60.4 \mathrm{kcal} \mathrm{mol}^{-1}\right) \mathrm{LC}$ (flat) conformation, the two $\mathrm{H}_{\text {amide }}$ atoms are at a similar $z$-distance from the surface (within $0.2 \AA)$. In this conformation, both of the $\mathrm{H}_{\text {amide }}$ atoms $\mathrm{H}$-bond with $\mathrm{O}_{\text {surf }}$ atoms, as shown in Fig. 3. In the LC (vertical) conformation, which has lower stability $\left(\mathrm{E}_{a d s}=-48.9 \mathrm{kcal} \mathrm{mol}^{-1}\right)$, two distinct $\mathrm{H}_{\text {amide }}-\mathrm{O}_{\text {surf }}$ distances are observed (separated by i, $1.0 \AA$ ). In this case, only one $\mathrm{H}_{\text {amide }}$ atom $\mathrm{H}$-bonds with a $\mathrm{O}_{\text {surf }}$ atom and the vdW interactions between the tailgroup atoms and the surface are much weaker. ${ }^{40}$ Two $\mathrm{H}_{\text {amide }}-\mathrm{O}_{\text {surf }}$ distances (separated by i. 1.0 $\AA)$ are also observed at $\mathrm{HC}\left(\mathrm{E}_{\text {ads }}=-51.7 \mathrm{kcal} \mathrm{mol}^{-1}\right)$; one $\mathrm{H}_{\text {amide }}$ atom $\mathrm{H}$-bonds with a $\mathrm{O}_{\text {surf }}$ atom, and the other which $\mathrm{H}$-bonds with the $\mathrm{O}_{\text {amide }}$ atom in a neighbouring molecule, as shown in Fig. 3. It is noteworthy that several almost isoenergetic conformations with slightly different $\mathrm{H}_{\text {amide }}-\mathrm{O}_{\text {surf }}$ distances were also observed in the DFT calculations; those included in the paramterization are shown in the SI (Fig. S1). In Fig. 6, the separation between the two $\mathrm{H}_{\text {amide }}$ peaks $\sim 1.0$ Åat all coverages for the Original FF. Collectively, this suggests that, when using the Original FF, the SAm headgroups adopt an intermediate conformation between LC (vertical) and LC (flat) (Fig. 2) from DFT, irregardless of the surface coverage.

Fig. 6 shows that the headgroup conformations are rather different when using the Optimized FF, particularly at MC and HC. The headgroup $\mathrm{C}$ atom peak is $\sim 0.4 \AA$ closer to the surface at all coverages studied for the Optimized FF compared to the Original FF, which corresponds to the shift in $z_{e q}$ shown in Fig. 4. At LC, there are two $\mathrm{H}_{\text {amide }}$ peaks (separated by $\sim 1.0 \AA$ ) and single $\mathrm{O}_{\text {amide }}$ and $\mathrm{N}_{\text {amide }}$ peaks, with the latter slightly $(\sim 0.5 \AA)$ closer to the surface. This suggests an intermediate between the LC (flat) and LC (vertical) conformations shown in Fig. 2 for the Optimized FF at LC, similar to the Original FF. Three separate $\mathrm{H}_{\text {amide }}$ peaks are observed in Fig. 6 at $\mathrm{MC}$ and $\mathrm{HC}$, the middle of which is the most intense and the latter two are separated by $i, 1.0 \AA$. There are also two $\mathrm{O}_{\text {amide }}$ and two 
$\mathrm{N}_{\text {amide }}$ peaks, with the first $\mathrm{O}_{\text {amide }}$ peak closest to the surface, followed by the first $\mathrm{N}_{\text {amide }}$ peak. This suggests that two different headgroup conformations are present when using the Optimized FF at MC and HC. One of these conformations is probably the most stable HC system shown in Fig. $2\left(\mathrm{E}_{a d s}=-51.7 \mathrm{kcal} \mathrm{mol}^{-1}\right)$ and the other the $\mathrm{HC}$ system shown in the SI (Fig. S1), which is slightly less stable $\left(\mathrm{E}_{a d s}=-50.5 \mathrm{kcal} \mathrm{mol}^{-1}\right)$. As the coverage increases, there are larger steric interactions between neighboring SAm molecules, which retards molecular rearrangement. Consequently, the SAm molecules are unable to rearrange themselves to reach the lowest $E_{a d s}$ conformations at $\mathrm{MC}$ and $\mathrm{HC}$, and can become trapped in geometries with slightly lower stability. In summary, the Optimized FF more faithfully reproduces the relaxed DFT conformations shown in Fig. 2 and Fig. S1 in large-scale NEMD simulations.

Fig. 7 shows atomic $x$-velocity profiles separated into $1.0 \AA$ spatial bins in the $z$ direction, $v_{x}(z)$. These are overlaid with SAm and $n$-hexadecane mass density profiles from 0.05 $\AA$ spatial bins in $z, \rho(z)$. Profiles for the top wall moving in the $+x$ direction are shown in Fig. 7, profiles for the top wall moving the $-x,+y$ and $-y$ directions are shown in the SI (Fig. S10-12). 


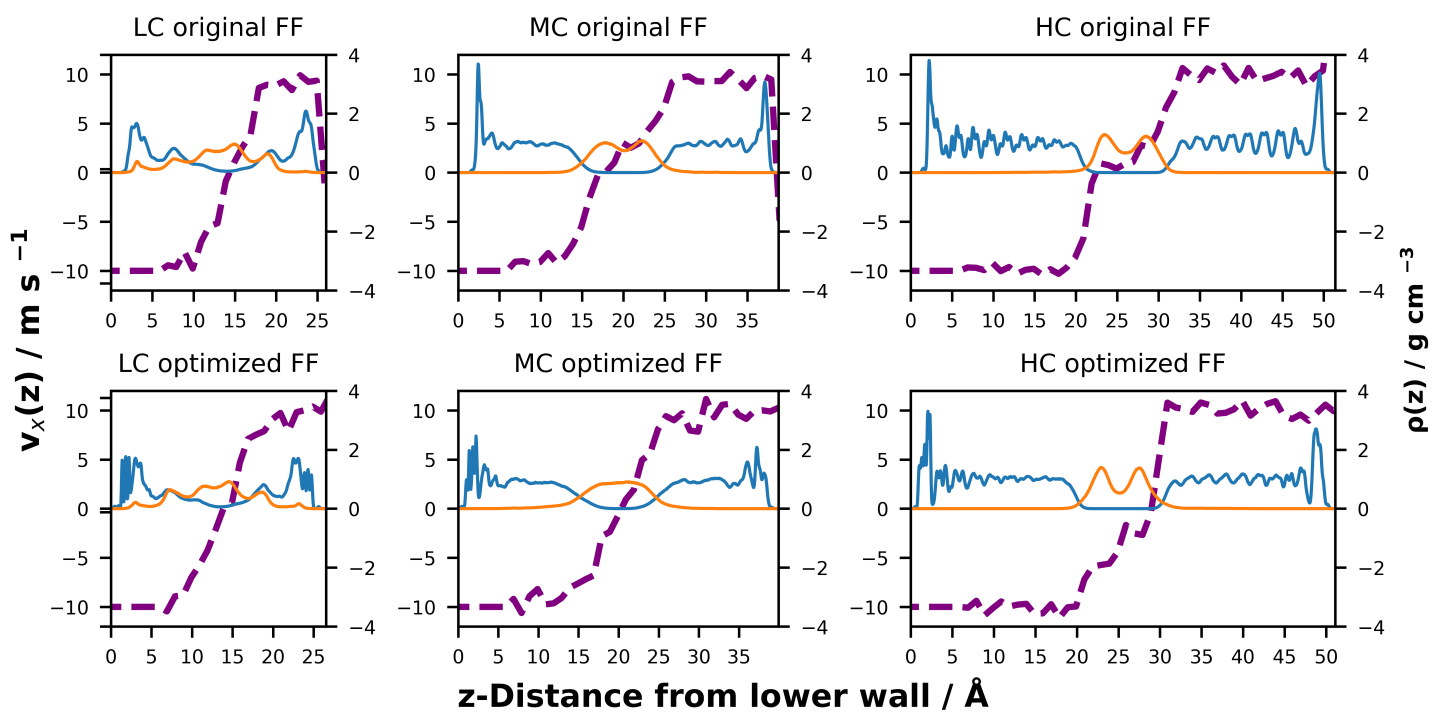

Figure 7: Velocity profiles for atomic velocities along the $x$ direction from $1.0 \AA$ spatial bins in $z, v_{x}(z)$. SAm and $n$-hexadecane mass density profiles from $0.05 \AA$ spatial bins in $z, \rho(z)$, are overlaid. Top wall moved in the $+x$ direction.

Fig. 7 suggests that, for all of the coverages studied, the structure and flow behaviour remains broadly similar for the Original FF and the Optimized FF. The mass density profiles show that the SAm films become thicker and show more layering with increasing coverage. Generally, fewer peaks are apparent when using the Optimized FF compared to the Original FF, suggesting less structured, more liquid-like films. ${ }^{19}$ The overlap between the SAm and $n$-hexadecane peaks decreases with increasing coverage, indicating less interdigitation. There is also slightly more interdigitation for the Optimized FF compared to the Original FF. Increased interdigitation has been shown to lead to higher friction in previous NEMD simulations of adsorbed surfactant films. ${ }^{19,84}$ Thus, purely from the mass density profiles, friction is expected to decrease with increasing coverage and to be slightly higher for the Optimized FF compared to the Original FF.

The velocity profiles indicate that the SAm headgroups move at the same velocity as the wall to which they are adsorbed. The tailgroups move at the same velocity as the headgroups until the outer region where they interdigitate with the $n$-hexadecane molecules, at which 
point they start to be sheared. ${ }^{19}$ At LC, $n$-hexadecane penetrates the entire film, so all but the first strongly adsorbed layer, i.e. the central $\sim 15$ Åis sheared. Interdigitation occurs $\sim$ 10 Åinto the MC film and the 10 Åthick central region is sheared. At HC, negligible shear occurs because slip planes form between the solid-like SAm films and the $n$-hexadecane lubricant. ${ }^{19,21}$ At all coverages, the velocity profiles are essentially indistinguishable between the Original FF and the Optimized FF, suggesting similar flow behaviour.

The friction behaviour was also compared between the Original FF and the Optimized FF, as shown in Fig. 8. The friction coefficient was calculated using the Amontons-Coulomb friction law under the high load approximation: $\mathrm{F}_{L} / \mathrm{F}_{N}=\mu+\mathrm{F}_{0} / \mathrm{F}_{N} \approx \mu$, where $\mathrm{F}_{L}$ and $\mathrm{F}_{N}$ are respectively the mean lateral (friction) force and normal force acting on the outer layer of atoms in each slab, and $\mathrm{F}_{0}$ is the load-independent Derjaguin offset representing adhesive surface forces. Previous NEMD simulations have confirmed the applicability of this approximation for OFM films between atomically-smooth surfaces with a separating lubricant layer. ${ }^{17-19}$ Points and error bars in Fig. 8 represent the mean and one standard deviation between the steady state friction coefficient from simulations with the top wall moving in the $+x,-x,+y,-y$ directions. This was to improve statistics as well as to account for potential friction anisotropy. ${ }^{81}$ 


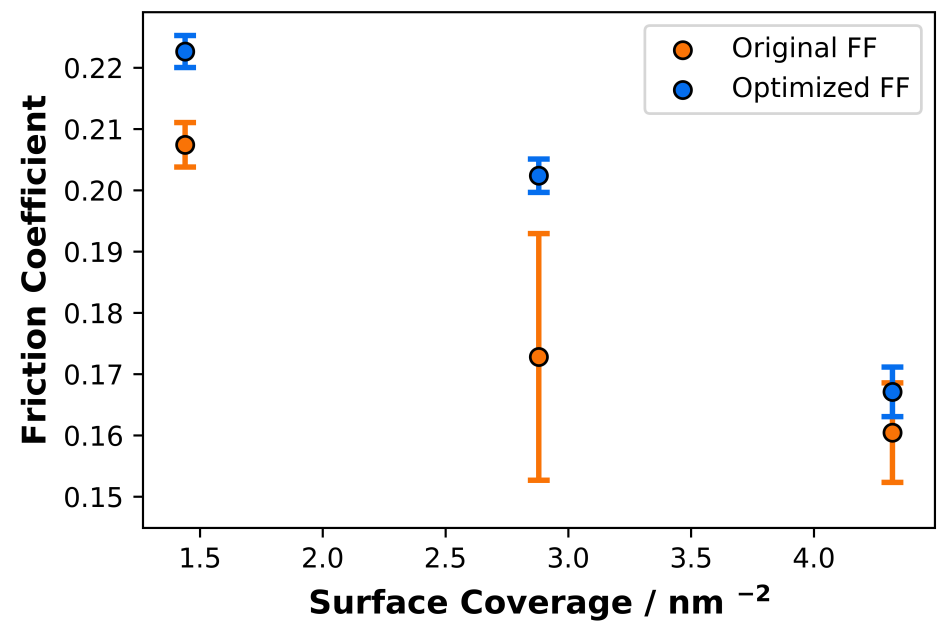

Figure 8: Change in the friction coefficient, $\mu$, with surface coverage for the Original FF compared to the Optimized FF. Circles and error bars represent the mean and one standard deviation between the steady state friction coefficient with the top wall moved the $+x,-x$, $+y$ and $-y$ directions.

For both the Original FF and the Optimized FF, the friction coefficient in Fig. 8 decreases approximately linearly with increasing surface coverage. This is in general agreement with previous NEMD simulations of surfactant-lubricated systems ${ }^{19,84}$ as well as atomic force microscopy experiments. ${ }^{85,86}$ In macroscopic friction tests, surfactant concentration rather than surface coverage is usually varied since this is much easier to measure and control. However, in boundary friction experiments ${ }^{15}$ surfactant molecules with saturated tailgroups, which have been shown to form higher coverage films than those containing $Z$-unsaturation, ${ }^{7}$ gave lower friction. Thus, the friction results in Fig. 8 are in qualitative agreement with trends from nanoscopic ${ }^{85,86}$ and macroscopic ${ }^{15}$ friction experiments.

The friction coefficients in Fig. 8 are all somewhat higher compared to those reported in Ref., ${ }^{19}$ but these are not directly comparable since the system and conditions are different. In Fig. 8, for all of the coverages studied, the mean friction coefficient is slightly higher for the Optimized FF compared to the Original FF; however, the difference is only statistically significant for the LC and MC cases. This is probably a consequence of the stronger amidesurface binding which leads to more disordered films and increased interdigitation. The 
similarity between the FFs is perhaps unsurprising given that the OFM films are preformed on the surfaces (similar to Langmuir-Blodgett experiments) ${ }^{78}$ and periodic in the lateral directions, which prevents molecules being squeezed out. ${ }^{76}$ Since the molecules cannot leave the contact, the stronger molecule-surface interactions only weakly affect the friction behaviour, which is dominated by the tailgroup interdigitation. ${ }^{19,84}$ Therefore, these simulations with the accurate Optimized FF validate the results of previous NEMD studies ${ }^{17-21}$ which used the Original $\mathrm{FF}^{34}$ derived from mixing rules and parameters developed for $n$-alkane adsorption. However, much larger differences between Original FF and Optimized FF are expected for other types of classical MD simulation. For example, the results of nanotribological simulations in which the surfactant molecules can be squeezed out of the contact will be more dependant upon the molecule-surface interaction strength. ${ }^{76,87}$ The molecule-surface interaction strength will also affect simulations of surfactant adsorption from nonpolar solvents. ${ }^{9,88}$ The Optimized FF developed here will facilitate MD simulations of amides on iron oxide surfaces in which the molecule-surface interactions faithfully reproduce interactions from accurate DFT calculations.

Unlike NEMD simulations of thiol monolayers adsorbed on gold surfaces, ${ }^{81}$ no systematic difference in the friction coefficient between the different sliding directions (friction asymmetry) was observed in these simulations. This is probably due to the thin lubricant layer which separates the OFM films. ${ }^{19}$ However, the smaller error bars in Fig. 8 indicate that there was less variation in the friction coefficient between the different sliding directions when the Optimized FF was used compared to the Original FF, particularly at MC.

\section{Conclusions}

In this study, an Optimized FF was developed for MD simulations of the interface between the amide surfactants and iron oxide surfaces. DFT $+U$ calculations using an accurate vdW functional were performed on a wide range of HAm geometries on $\alpha-\mathrm{Fe}_{2} \mathrm{O}_{3}(0001)$ to 
ensure extensive sampling of the conformational phase space. The polar amide headgroups strongly chemisorb on the surface and it was found that these interactions could not be accurately represented with a standard LJ potential. Instead, a Morse potential was used for the most strongly interacting molecule-surface atom pairs; $\mathrm{O}_{\text {amide }}-\mathrm{Fe}_{\text {surf }}, \mathrm{N}_{\text {amide }}-\mathrm{Fe}_{\text {surf }}$, and $\mathrm{H}_{\text {amide }}-\mathrm{O}_{\text {surf }}$. Different optimization algorithms and constraints were tested, which ultimately yielded excellent agreement between the Optimized FF and the DFT calculations.

The Optimized FF was tested in large-scale NEMD simulations of a thin layer of $n$ hexadecane lubricant confined between amide films with different coverages adsorbed on iron oxide surfaces. The amide molecules were drawn closer to the surface and the orientation of the headgroup was more similar to that observed in the DFT calculations when the Optimized FF was employed. However, for the systems and conditions studied, the velocity profiles and friction coefficient are very similar for the Original FF and the Optimized FF. Since the molecules cannot be squeezed out of the contact, the stronger molecule-surface interactions do not significantly affect the friction behaviour, which is dominated by the tailgroup interdigitation. Therefore, these results validate the findings of previous NEMD studies which used the Original FF derived from mixing rules and parameters developed for $n$-alkane adsorption. The Optimized FF will facilitate MD simulations at the amide-iron oxide interface in which the interactions are representative of those observed in accurate DFT calculations.

\section{Acknowledgement}

The authors thank Hongyu Gao, Martin H. Müser, Joshua D. Moore and Joseph Remias for useful discussions. C. A. L. thanks Afton Chemical Corp. and the Engineering and Physical Sciences Research Council (EPSRC) for PhD funding via the Theory and Simulation of Materials-Center for Doctoral Training (TSM-CDT) EP/L015579/1. J. P. E. and D. D. acknowledge the EPSRC for Established Career Fellowship EP/N025954/1 and grant 
EP/P030211/1. C. G. is supported by the European Union's Horizon 2020 research and innovation program under the Marie Skodowska-Curie grant agreement No. 744027. We acknowledge the use of Imperial College London Research Computing Service (RCS). All data reported in the manuscript can be obtained by emailing the corresponding author or tribology@imperial.ac.uk.

\section{Supporting Information Available}

Supporting Information (SI) includes; additional relaxed DFT configurations, details of the force-field parameterization algorithm, comparisons of the performance different functional forms, tests for $n$-alkane adsorption, and additional results from the molecular dynamics simulations.

This material is available free of charge via the Internet at http://pubs.acs.org/.

\section{References}

(1) Heinz, H.; Pramanik, C.; Heinz, O.; Ding, Y.; Mishra, R. K.; Marchon, D.; Flatt, R. J.; Estrela-Lopis, I.; Llop, J.; Moya, S.; Ziolo, R. F. Nanoparticle decoration with surfactants: molecular interactions, assembly, and applications. Surf. Sci. Rep. 2017, 72, $1-58$.

(2) Araujo, A. C.; Viana, P. R. M.; Peres, A. E. C. Reagents in iron ores flotation. Miner. Eng. 2005, 18, 219-224.

(3) Zhu, Y.; Free, M. L.; Woollam, R.; Durnie, W. A review of surfactants as corrosion inhibitors and associated modeling. Prog. Mater. Sci. 2017, 90, 159-223.

(4) Spikes, H. Friction Modifier Additives. Tribol. Lett. 2015, 60, 5. 
(5) Holmberg, K.; Erdemir, A. Influence of tribology on global energy consumption, costs and emissions. Friction 2017, 5, 263-284.

(6) Campen, S.; Green, J. H.; Lamb, G. D.; Spikes, H. A. In Situ Study of Model Organic Friction Modifiers Using Liquid Cell AFM; Saturated and Mono-unsaturated Carboxylic Acids. Tribol. Lett. 2015, 57, 18.

(7) Wood, M. H.; Casford, M. T.; Steitz, R.; Zarbakhsh, A.; Welbourn, R. J. L.; Clarke, S. M. Comparative Adsorption of Saturated and Unsaturated Fatty Acids at the Iron Oxide/Oil Interface. Langmuir 2016, 32, 534.

(8) Oh, S. J.; Cook, D. C.; Townsend, H. E. Characterization of iron oxides commonly formed as corrosion products on steel. Hyperfine Interact. 1998, 112, 59-66.

(9) Jaishankar, A.; Jusufi, A.; Vreeland, J. L.; Deighton, P.; Pellettiere, J. R.; Schilowitz, A. M. Adsorption of stearic acid at the iron oxide/oil interface - theory, experiments and modeling. Langmuir 2019, 35, 2033-2046.

(10) Sahoo, R. R.; Biswas, S. K. Frictional response of fatty acids on steel. J. Colloid Interface Sci. 2009, 333, 707-718.

(11) Chernyshova, I. V.; Ponnurangam, S.; Somasundaran, P. Adsorption of Fatty Acids on Iron (Hydr)oxides from Aqueous Solutions. Langmuir 2011, 27, 10007-10018.

(12) Wood, M. H.; Welbourn, R. J. L.; Charlton, T.; Zarbakhsh, A.; Casford, M. T.; Clarke, S. M. Hexadecylamine Adsorption at the Iron Oxide-Oil Interface. Langmuir 2013, 29, 13735-13742.

(13) Loehlé, S.; Matta, C.; Minfray, C.; Le Mogne, T.; Iovine, R.; Obara, Y.; Miyamoto, A.; Martin, J.-M. Mixed lubrication of steel by C18 fatty acids revisited. Part I: toward the formation of carboxylate. Tribol. Int. 2015, 82, 218-227. 
(14) Ruths, M.; Lundgren, S.; Danerlov, K.; Persson, K. Friction of fatty acids in nanometersized contacts of different adhesive strength. Langmuir 2008, 24, 1509-1516.

(15) Campen, S.; Green, J.; Lamb, G.; Atkinson, D.; Spikes, H. On the increase in boundary friction with sliding speed. Tribol. Lett. 2012, 48, 237-248.

(16) Ewen, J. P.; Heyes, D. M.; Dini, D. Advances in nonequilibrium molecular dynamics simulations of lubricants and additives. Friction 2018, 6, 349-386.

(17) Doig, M.; Warrens, C. P.; Camp, P. J. Structure and friction of stearic acid and oleic acid films adsorbed on iron oxide surfaces in squalane. Langmuir 2014, 30, 186-195.

(18) Doig, M.; Camp, P. J. The structures of hexadecylamine films adsorbed on iron-oxide surfaces in dodecane and hexadecane. Phys. Chem. Chem. Phys. 2015, 17, 5248-5255.

(19) Ewen, J. P.; Gattinoni, C.; Morgan, N.; Spikes, H. A.; Dini, D. Nonequilibrium Molecular Dynamics Simulations of Organic Friction Modifiers Adsorbed on Iron Oxide Surfaces. Langmuir 2016, 32, 4450.

(20) Ewen, J. P.; Gattinoni, C.; Thakkar, F. M.; Morgan, N.; Spikes, H.; Dini, D. A Comparison of Classical Force-Fields for Molecular Dynamics Simulations of Lubricants. Materials. 2016, 9, 651 .

(21) Ewen, J. P.; Kannam, S. K.; Todd, B. D.; Dini, D. Slip of alkanes confined between surfactant monolayers adsorbed on solid surfaces. Langmuir 2018, 34, 3864-3873.

(22) Allen, W.; Rowley, R. L. Predicting the viscosity of alkanes using nonequilibrium molecular dynamics: Evaluation of intermolecular potential models. J. Chem. Phys. 1997, 106, 10273-10281.

(23) Harrison, J. A.; Schall, J. D.; Maskey, S.; Mikulski, P. T.; Knippenberg, M. T.; Morrow, B. H.; Harrison, J. A.; Schall, J. D.; Maskey, S.; Mikulski, P. T.; Knippen- 
berg, M. T.; Morrow, B. H. Review of force fields and intermolecular potentials used in atomistic computational materials research. Appl. Phys. Rev. 2018, 5, 031104.

(24) Jorgensen, W. L.; Maxwell, D. S.; Tirado-Rives, J. Development and testing of the OPLS all-atom force field on conformational energetics and properties of organic liquids. J. Am. Chem. Soc. 1996, 118, 11225-11236.

(25) Price, M. L. P.; Ostrovsky, D.; Jorgensen, W. L. Gas-phase and liquid-state properties of esters, nitriles, and nitro compounds with the OPLS-AA force field. J. Comput. Chem. 2001, 22, 1340-1352.

(26) Siu, S. W. I.; Pluhackova, K.; Bockmann, R. A. Optimization of the OPLS-AA Force Field for Long Hydrocarbons. J. Chem. Theory Comput. 2012, 8, 1459-1470.

(27) Wang, J. M.; Wolf, R. M.; Caldwell, J. W.; Kollman, P. A.; Case, D. A. Development and testing of a general amber force field. J. Comput. Chem. 2004, 25, 1157-1174.

(28) Vanommeslaeghe, K.; Hatcher, E.; Acharya, C.; Kundu, S.; Zhong, S.; Shim, J.; Darian, E.; Guvench, O.; Lopes, P.; Vorobyov, I.; MacKerell, A. D. CHARMM General Force Field: A Force Field for Drug-Like Molecules Compatible with the CHARMM All-Atom Additive Biological Force Fields. J. Comput. Chem. 2010, 31, 671-690.

(29) Sun, H.; Jin, Z.; Yang, C.; Akkermans, R. L.; Robertson, S. H.; Spenley, N. A.; Miller, S.; Todd, S. M. COMPASS II: extended coverage for polymer and drug-like molecule databases. J. Mol. Model. 2016, 22, 1-10.

(30) Herbers, C. R.; Li, C.; van der Vegt, N. F. A. Grand Challenges in Quantum-Classical Modeling of Molecule- Surface Interactions. J. Comput. Chem. 2013, 34, 1177-1188.

(31) Lennard-Jones, J. E. On the determination of molecular fields.-II. From the equation of state of a gas. Proc. R. Soc. Lond. A 1924, 106, 463-477. 
(32) Heinz, H.; Lin, T. J.; Kishore Mishra, R.; Emami, F. S. Thermodynamically consistent force fields for the assembly of inorganic, organic, and biological nanostructures: The INTERFACE force field. Langmuir 2013, 29, 1754-1765.

(33) Cygan, R. T.; Liang, J. J.; Kalinichev, A. G. Molecular models of hydroxide, oxyhydroxide, and clay phases and the development of a general force field. J. Phys. Chem. $B$ 2004, 108, 1255-1266.

(34) Berro, H.; Fillot, N.; Vergne, P. Molecular dynamics simulation of surface energy and ZDDP effects on friction in nano-scale lubricated contacts. Tribol. Int. 2010, 43, 18111822.

(35) Savio, D.; Fillot, N.; Vergne, P.; Zaccheddu, M. A Model for Wall Slip Prediction of Confined n-Alkanes: Effect of Wall-Fluid Interaction Versus Fluid Resistance. Tribol. Lett. 2012, 46, 11-22.

(36) Carrasco, J.; Liu, W.; Michaelides, A.; Tkatchenko, A. Insight into the description of van der Waals forces for benzene adsorption on transition metal (111) surfaces. J. Chem. Phys. 2014, 140, 084704.

(37) Murphy, C. J.; Carrasco, J.; Lawton, T. J.; Liriano, M. L.; Baber, A. E.; Lewis, E. A.; Michaelides, A.; Sykes, E. C. H. Structure and energetics of hydrogen-bonded networks of methanol on close packed transition metal surfaces. J. Chem. Phys. 2014, 141, 014701.

(38) Bedolla, P. O.; Feldbauer, G.; Wolloch, M.; Eder, S. J.; Dorr, N.; Mohn, P.; Redinger, J.; Vernes, A. Effects of van der Waals Interactions in the Adsorption of Isooctane and Ethanol on Fe(100) Surfaces. J. Phys. Chem. C 2014, 118, 17608-17615.

(39) Gattinoni, C.; Michaelides, A. Understanding corrosion inhibition with van der Waals DFT methods: The case of benzotriazole. Faraday Discuss. 2015, 180, 439-458. 
(40) Gattinoni, C.; Ewen, J. P.; Dini, D. Adsorption of Surfactants on $\alpha$-Fe2O3(0001): A Density Functional Theory Study. J. Phys. Chem. C 2018, 122, 20817-20826.

(41) Larrucea, J.; Lid, S.; Colombi Ciacchi, L. Parametrization of a classical force field for iron oxyhydroxide/water interfaces based on Density Functional Theory calculations. Comput. Mater. Sci. 2014, 92, 343-352.

(42) Ta, T. D.; Tieu, A. K.; Zhu, H.; Kosasih, B. Adsorption of Normal-Alkanes on Fe(110), FeO(110), and Fe2O3(0001): Influence of Iron Oxide Surfaces. J. Phys. Chem. C 2015, 119, 12999-13010.

(43) Tromp, S.; Joly, L.; Cobian, M.; Fillot, N. Chemical Physics at Interfaces within a Refrigerant-Lubricated Contact: From Electronic Structure to Large-Scale Molecular Dynamics Simulations. J. Phys. Chem. C 2018, 122, 5420-5429.

(44) Kresse, G.; Hafner, J. Ab initio molecular dynamics for liquid metals. Phys. Rev. B 1993, $47,558$.

(45) Kresse, G.; Furthmuller, J. Efficiency of ab-initio total energy calculations for metals and semiconductors using a plane-wave basis set. Comput. Mater. Sci. 1996, 6, 15-50.

(46) Kresse, G.; Furthmuller, J. Efficient iterative schemes for ab initio total-energy calculations using a plane-wave basis set. Phys. Rev. B 1996, 54, 11169.

(47) Kresse, G.; Joubert, D. From ultrasoft pseudopotentials to the projector augmentedwave method. Phys. Rev. B. 1999, 59, 1758-1775.

(48) Klimeš, J.; Bowler, D. R.; Michaelides, A. Chemical accuracy for the van der Waals density functional. J. Phys. Condens. Matter 2010, 22.

(49) Klimeš, J.; Bowler, D. R.; Michaelides, A. Van der Waals density functionals applied to solids. Phys. Rev. B 2011, 83, 195131. 
(50) Dudarev, S.; Botton, G. Electron-energy-loss spectra and the structural stability of nickel oxide: An LSDA+U study. Phys. Rev. B 1998, 57, 1505-1509.

(51) Huang, X.; Ramadugu, S. K.; Mason, S. E. Surface-Specific DFT + U Approach Applied to $\alpha$-Fe2O3(0001). J. Phys. Chem. C 2016, 120, 4919-4930.

(52) Makov, G.; Payne, M. Periodic boundary conditions in ab initio calculations. Phys. Rev. B 1995, 51, 4014-4022.

(53) Humphrey, W.; Dalke, A.; Schulten, K. VMD: Visual molecular dynamics. J. Mol. Graph. Model. 1996, 14, 33-38.

(54) Parkinson, G. S. Iron oxide surfaces. Surf. Sci. Rep. 2016, 71, 272-365.

(55) Yamamoto, S.; Kendelewicz, O. T.; Newberg, J. T.; Ketteler, G.; Starr, D. E.; Mysak, E. R.; Andersson, K. J.; Ogasawara, H.; Bluhm, H.; Salmeron, M.; Brown, G. E.; Nilsson, A. Water Adsorption on a-Fe2O3 (0001) at near Ambient Conditions. J. Phys. Chem. C 2010, 114, 2256-2266.

(56) Lancaster, J. K. A review of the influence of environmental humidity and water on friction, lubrication and wear. Tribol. Int. 1990, 23, 371-389.

(57) Wilson, D.; Langell, M. A. XPS analysis of oleylamine/oleic acid capped Fe3O4 nanoparticles as a function of temperature. Appl. Surf. Sci. 2014, 303, 6-13.

(58) Filius, J. D.; Hiemstra, T.; Riemsdijk, W. H. V. Adsorption of Small Weak Organic Acids on Goethite: Modeling of Mechanisms. J. Colloid Interface Sci. 1997, 380, 368380.

(59) Tsuzuki, S.; Honda, K.; Uchimaru, T.; Mikami, M. Magnitude of interaction between nalkane chains and its anisotropy: High-level ab initio calculations of n-butane, n-petane, and n-hexane dimers. J. Phys. Chem. A 2004, 108, 10311-10316. 
(60) Gao, D. Z.; Federici Canova, F.; Watkins, M. B.; Shluger, A. L. Efficient parametrization of complex molecule-surface force fields. J. Comput. Chem. 2015, 36, 1187-1195.

(61) Huan, T. D.; Batra, R.; Chapman, J.; Krishnan, S.; Chen, L.; Ramprasad, R. A universal strategy for the creation of machine learning-based atomistic force fields. $n p j$ Comput. Mater. 2017, 3, 37.

(62) Johnston, K.; Herbers, C. R.; Van Der Vegt, N. F. Development of classical moleculesurface interaction potentials based on density functional theory calculations: Investigation of force field representability. J. Phys. Chem. C 2012, 116, 19781-19788.

(63) Bamdad, M.; Farrokhpour, H.; Ashrafizaadeh, M. A new force field for the adsorption of $\mathrm{H} 2, \mathrm{O} 2, \mathrm{~N} 2, \mathrm{CO}, \mathrm{H} 2 \mathrm{O}$, and $\mathrm{H} 2 \mathrm{~S}$ gases on alkali doped carbon nanotubes. Mol. Phys. 2016, 114, 3375-3387.

(64) Berg, A.; Peter, C.; Johnston, K. Evaluation and Optimization of Interface Force Fields for Water on Gold Surfaces. J. Chem. Theory Comput. 2017, 13, 5610-5623.

(65) Bolton, K.; Bosio, S.; Hase, W. L.; Schneider, W. F.; Hass, K. C. Comparison of Explicit and United Atom Models for Alkane Chains Physisorbed on $\alpha$-Al2O3 (0001). J. Phys. Chem. B 1999, 103, 3885-3895.

(66) Plimpton, S. Fast Parallel Algorithms for Short-Range Molecular Dynamics. J. Comput. Phys. 1995, 117, $1-19$.

(67) Yeh, I. C.; Berkowitz, M. L. Ewald summation for systems with slab geometry. $J$. Chem. Phys. 1999, 111, 3155-3162.

(68) Poli, R.; Kennedy, J.; Blackwell, T. Particle swarm optimization: An overview. Swarm Intell. 2007, 1, 33-57.

(69) Wang, L.-P.; Chen, J.; Van Voorhis, T. Systematic Parametrization of Polarizable Force Fields from Quantum Chemistry Data. J. Chem. Theory Comput. 2013, 9, 452-460. 
(70) Kong, L. T.; Denniston, C.; Müser, M. H.; Qi, Y. Non-bonded force field for the interaction between metals and organic molecules: a case study of olefins on aluminum. Phys. Chem. Chem. Phys. 2009, 11, 10195-10203.

(71) Ercolessi, F.; Adams, J. Interatomic Potentials From First-Principles Calculations: The Force-Matching Method. Europhys. Lett. 1994, 26, 583-588.

(72) Morse, P. Diatomic Molecules According to the Wave Mechanics. II. Vibrational Levels. Phys. Rev. 1929, 34, 57.

(73) Mahaffy, R.; Bhatia, R.; Garrison, B. J. Diffusion of a Butanethiolate Molecule on a Au\{111\} Surface. J. Phys. Chem. B 1997, 101, 771-773.

(74) Ahn, Y.; Saha, J. K.; Schatz, G. C.; Jang, J. Molecular Dynamics Study of the Formation of a Self-Assembled Monolayer on Gold. J. Phys. Chem. C 2011, 115, 10668-10674.

(75) Dixon, D. A.; Dobbs, K. D.; Valentini, J. J. Amide-Water and Amide-Amide Hydrogen Bond Strengths. J. Phys. Chem. 1994, 98, 13435-13439.

(76) Tartaglino, U.; Sivebaek, I. M.; Persson, B. N. J.; Tosatti, E. Impact of molecular structure on the lubricant squeeze-out between curved surfaces with long range elasticity. $J$. Chem. Phys. 2006, 125, 014704.

(77) Ryckaert, J. P.; Ciccotti, G.; Berendsen, H. J. C. Numerical-integration of Cartesian equations of motion of a system with constraints: molecular-dynamics of n-alkanes. $J$. Comput. Phys. 1977, 23, 327-341.

(78) Briscoe, B. J.; Evans, D. C. B. The shear properties of Langmuir-Blodgett layers. Proc. R. Soc. Lond. A 1982, 380, 389.

(79) Ramachandran, S.; Tsai, B. L.; Blanco, M.; Chen, H.; Tang, Y. C.; Goddard, W. A. Self-assembled monolayer mechanism for corrosion inhibition of iron by imidazolines. Langmuir 1996, 12, 6419-6428. 
(80) Schneider, T.; Stoll, E. Molecular-dynamics study of a three-dimensional onecomponent model for distortive phase-transitions. Phys. Rev. B 1978, 17, 1302-1322.

(81) Jabbarzadeh, A. Friction anisotropy and asymmetry in self assembled monolayers. Tribol. Int. 2016, 102, 600-607.

(82) Bernardi, S.; Todd, B. D.; Searles, D. J. Thermostating highly confined fluids. J. Chem. Phys. 2010, 132, 244706.

(83) O'Connor, T. C.; Andzelm, J.; Robbins, M. O. AIREBO-M: A reactive model for hydrocarbons at extreme pressures. J. Chem. Phys. 2015, 142, 024903.

(84) Kong, Y. C.; Tildesley, D. J.; Alejandre, J. The molecular dynamics simulation of boundary-layer lubrication. Mol. Phys. 1997, 92, 7-18.

(85) Lee, S.; Shon, Y.-S.; Colorado, R.; Guenard, R. L.; Lee, T. R.; Perry, S. S. The Influence of Packing Densities and Surface Order on the Frictional Properties of Alkanethiol SelfAssembled Monolayers (SAMs) on Gold: A Comparison of SAMs Derived from Normal and Spiroalkanedithiols. Langmuir 2000, 2220-2224.

(86) Flater, E. E.; Ashurst, W. R.; Carpick, R. W. Nanotribology of octadecyltrichlorosilane monolayers and silicon: Self-mated versus unmated interfaces and local packing density effects. Langmuir 2007, 23, 9242-9252.

(87) Summers, A. Z.; Iacovella, C. R.; Cummings, P. T.; McCabe, C. Investigating Alkylsilane Monolayer Tribology at a Single-Asperity Contact with Molecular Dynamics Simulation. Langmuir 2017, 33, 11270-11280.

(88) Bradley-Shaw, J. L.; Camp, P. J.; Dowding, P. J.; Lewtas, K. Molecular Dynamics Simulations of Glycerol Monooleate Confined between Mica Surfaces. Langmuir 2016, 32, $7707-7718$. 
Graphical TOC Entry

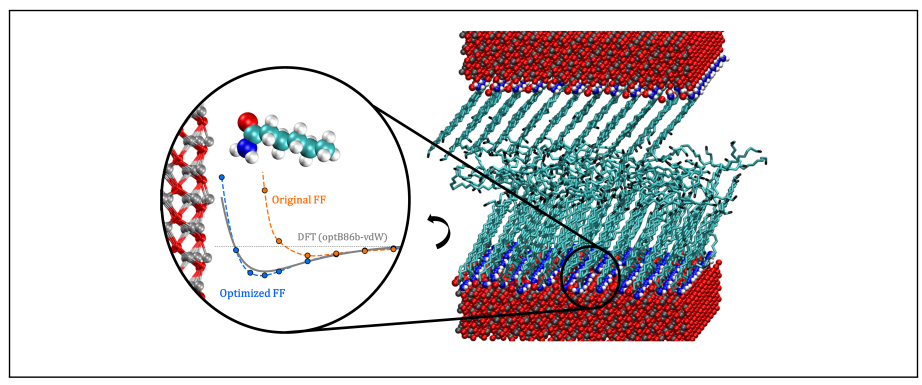




\section{Supporting Information for:}

\section{Simulating Surfactant-Iron Oxide Interfaces:}

From Density Functional Theory to Molecular

\section{Dynamics}

Carlos Ayestarán Latorre, ${ }^{\dagger} \uparrow$ James P. Ewen, ${ }^{*, \dagger}$, Chiara Gattinoni, ${ }^{\ddagger}, \dagger$ and Daniele Dini ${ }^{\dagger}$

$\dagger$ Department of Mechanical Engineering, Imperial College London, London SW7 2AZ, UK

$\ddagger$ Department of Materials, ETH Zürich, Zürich 8092, Switzerland

I These authors contributed equally to this work

E-mail: j.ewen@imperial.ac.uk 


\section{Additional relaxed DFT configurations}

Additional relaxed DFT conformations of HAm adsorbed on $\alpha-\mathrm{Fe}_{2} \mathrm{O}_{3}(0001)$ at $\mathrm{HC}$ and LC, which were not shown in Gattinoni et al. $^{1}$ or the main text are provided in Fig. S1. Top views of these conformations are shown the associated $E_{a d s}$ values are given in the caption. These conformations, with slightly lower stability, were included in the parameterization to increase the training dataset and reduce overfitting. ${ }^{2,3}$

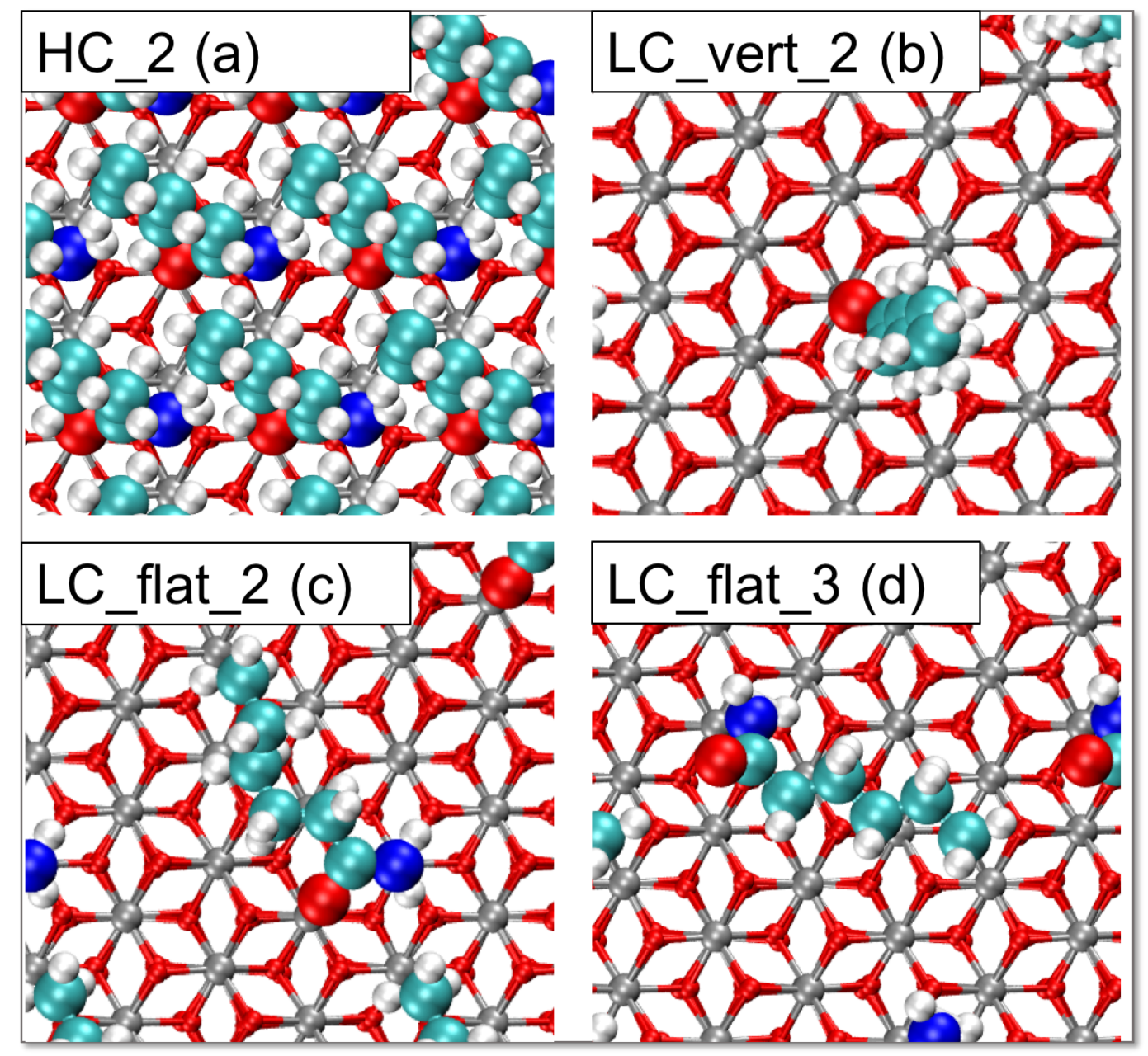

Figure S1: Top views of additional relaxed DFT configurations of HAm adsorbed on $\alpha$ $\mathrm{Fe}_{2} \mathrm{O}_{3}(0001)$ included in the fitting; $E_{a d s}=-50.5 \mathrm{kcal} \mathrm{mol}^{-1}$ (a), $-47.5 \mathrm{kcal} \mathrm{mol}^{-1}$ (b), -59.5 kcal $\mathrm{mol}^{-1}(\mathrm{c})$, and $-47.5 \mathrm{kcal} \mathrm{mol}^{-1}(\mathrm{~d})$.

Since $n$-alkane molecules are also included in the large-scale NEMD simulations, $E_{a d s}$ was also calculated for $n$-hexane on $\alpha-\mathrm{Fe}_{2} \mathrm{O}_{3}(0001)$ using DFT. A top view of the most 
stable conformation and the associated $E_{a d s}$ are shown in Fig. S2. These results were not included in the parameterization but are used to ensure that the Optimized FF gives similar performance to the Original FF for $n$-alkane adsorption.

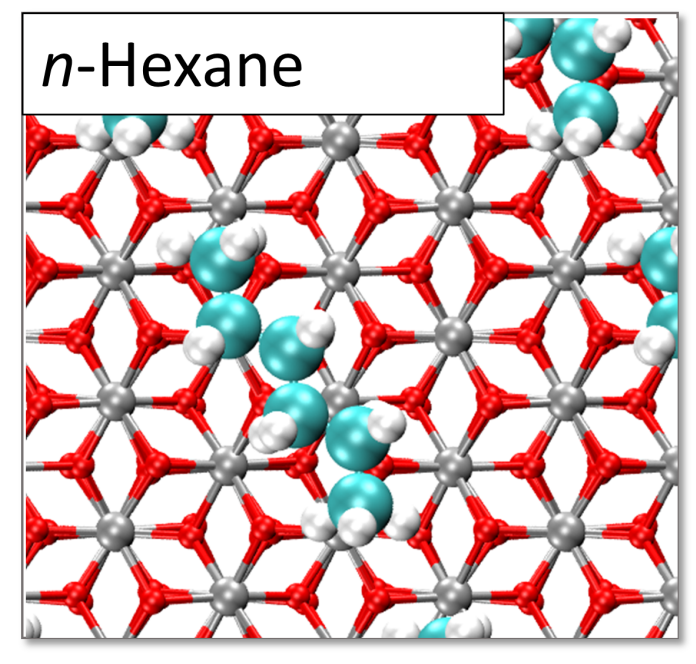

Figure S2: Top views of relaxed DFT configurations of $n$-hexane adsorbed on $\alpha-\mathrm{Fe}_{2} \mathrm{O}_{3}(0001)$; $E_{a d s}=-28.4 \mathrm{kcal} \mathrm{mol}^{-1}$

\section{Performance of the Original FF}

DFT calculations have been used to parameterize interface FFs for the interactions between several molecules and iron oxide surfaces. For example, Tromp et al. ${ }^{4}$ developed parameters for the 12-6 LJ and Coulombic potentials to match $E_{a d s}$ from DFT calculations for R1233zd(E) refrigerant molecules relaxed on a $\alpha-\mathrm{Fe}_{2} \mathrm{O}_{3}(01 \overline{1} 2)$ surface. For the interactions between the refrigerant molecules, they used a FF developed by Raabe and Maginn. ${ }^{5}$ Instead of using standard parameters for the surface atoms and relying upon mixing rules, Tromp et al. parameterized all of the individual interactions for optimal agreemtent with the DFT results. ${ }^{4}$ Ta et al. ${ }^{6}$ developed an interface $\mathrm{FF}$ for the interaction between $n$-alkanes and $\mathrm{Fe}(110), \mathrm{FeO}(110)$, and $\mathrm{Fe}_{2} \mathrm{O}_{3}$ (0001) surfaces from DFT calculations using a trial-and-error

approach. They employed the COMPASS-FF for the $n$-alkane molecules ${ }^{7,8}$ which includes a 9-6 LJ potential for the non-bonded interactions. ${ }^{6}$ A 9-6 LJ potential was also used for the 
$n$-alkane-surface interactions. However, the DFT calculations did not include a $U$ correction, so an inaccurate band gap for $\mathrm{Fe}_{2} \mathrm{O}_{3}$ and thus $E_{a d s}$ is to be expected. ${ }^{9}$ In any case, neither of these FFs, ${ }^{4,6}$ nor the INTERFACE-FF ${ }^{10}$ are directly transferable to the system of interest here.

To the authors' knowledge, the only FFs which are readily transferable to amide-iron oxide systems are ClayFF ${ }^{11}$ and the FFs developed by Berro et al. ${ }^{12}$ and Savio et al. ${ }^{13}$ Indeed, these FFs have been used in a number of previous MD simulations of similar systems. ${ }^{14-19}$ Berro et al. ${ }^{12}$ used the OPLS united-atom $\mathrm{FF}^{20}$ for the $n$-hexadecane molecules and the standard OPLS parameters for the $\mathrm{O}_{\text {surf }}$ atoms. Parameters were tested for 12-6 LJ and Coulombic potentials. The $\epsilon_{F e}$ parameter was varied between $0.5-15 \times \epsilon_{O}$ in order to investigate a range of interaction strengths. An intermediate value of $\epsilon_{F e}\left(2.5 \times \epsilon_{O}\right)$ used by Berro al. ${ }^{12}$ provided the best agreement with the current DFT calculations, so this was used in the remainder of this study. Savio et al. ${ }^{13}$ refined these 12-6 LJ parameters in order to yield realistic desorption energies for $\mathrm{CH}_{2}$ and $\mathrm{CH}_{3}$ segments on $\mathrm{Fe}_{2} \mathrm{O}_{3}$ surfaces. However, they did not include partial charge parameters, since the $n$-alkane molecules of interest were non-polar. ClayFF ${ }^{11}$ was developed to model the interactions between various metal oxides/hydroxides and water. Compared to the other FFs considered, the $\epsilon$ values are relatively small, but the partial charges are large meaning that the interactions are dominated by the electrostatics. The parameters from $\mathrm{ClayFF}^{11}$ as well as those developed by Berro al. ${ }^{12}$ and Savio et al. ${ }^{13}$ as used here are shown in Table S1.

Fig. S3 shows that all of the existing FFs overestimated $z_{e q}$ and underestimated $E_{a d s}$ for the amide-iron oxide system compared to he DFT calculations. The FF developed by Berro al. ${ }^{12}$ was selected as the 'Original FF' since it included partial charge parameters and facilitated slightly better agreement the DFT results, as shown in Fig. S3. 
Table S1: Surface LJ and Coulomb parameters developed by Berro et al. ${ }^{12}$ with and Savio et al. ${ }^{13}$

\begin{tabular}{lccc}
\hline & $\epsilon / \mathrm{kcal} \mathrm{mol}^{-1}$ & $\sigma / \AA$ & $q / \mathrm{e}$ \\
ClayFF $^{11}$ & 0.000009 & 6.181 & 1.575 \\
$\mathrm{Fe}_{\text {surf }}$ & 0.155 & 3.988 & -1.050 \\
$\mathrm{O}_{\text {surf }}$ & $\epsilon / \mathrm{kcal} \mathrm{mol}^{-1}$ & $\sigma / \AA$ & $q / \mathrm{e}$ \\
\hline & & & \\
Berro et al. $^{12}$ & 0.425 & 2.200 & 0.771 \\
Fe $_{\text {surf }}$ & 0.170 & 2.960 & -0.514 \\
$\mathrm{O}_{\text {surf }}$ & & & \\
\hline & $\epsilon / \mathrm{kcal} \mathrm{mol}^{-1}$ & $\sigma / \AA$ & $q / \mathrm{e}$ \\
Savio et al. $^{13}$ & & & \\
$\mathrm{Fe}_{\text {surf }}$ & 9.518 & 2.321 & - \\
$\mathrm{O}_{\text {surf }}$ & 0.210 & 2.960 & - \\
\hline
\end{tabular}
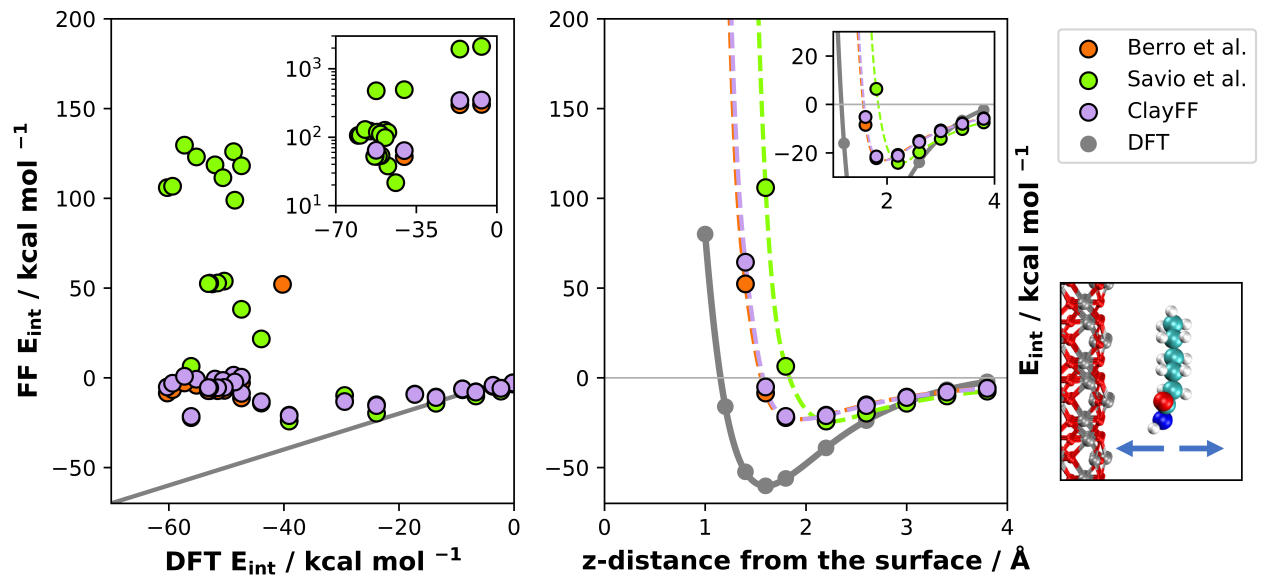

Figure S3: Performance of the interfacial FF employed by Berro et al. ${ }^{12}$ and Savio et al. ${ }^{13}$ compared to DFT calculations. (a) Comparison of $\mathrm{E}_{\text {int }}$, (b) z-scans of $\mathrm{E}_{\text {int }}$ for the flat LC HAm configuration. Lines are spline interpolations to the data.

\section{Force-field parameterization algorithm}

The classical FF parameters, $k$, for the surface and molecules atom pairs (e.g., $\epsilon_{i j}, \sigma_{i j}, q_{i j}$ for a $\mathrm{LJ}+\mathrm{C}$ potential) were tuned to match the DFT-obtained values. The aim of the FF 
parameterization was thus to minimize the objective function:

$$
\chi^{2}(k ; w)=\sum_{g=1}^{N_{G}} \sum_{v=1}^{N_{V}} w_{g, v}\left|m_{g, v}(k)-m_{g, v}^{0}\right|^{2}+\sum_{i=1}^{N_{P}} w_{i}\left|k_{i}-k_{i}^{0}\right|^{2}
$$

where $N_{G}$ is the number of available geometries and $N_{V}$ the number of values to match per geometry; $m_{g}^{0}$ are quantities obtained from DFT; $m_{g}(k)$ are the corresponding FF values for a given parametrization, $w_{g, v}$ are the weights associated with each geometry and value. In this study, we employed interaction energies $E_{\text {int }}$ and net forces $F_{\text {int }}$ between the molecules and the surface as the quantities to match. The $F_{\text {int }}$ values were only matched for the relaxed DFT configurations, where the expected values are zero. The second summation is a regularization term that provides a set of constrains to penalize large deviations from original parameter guesses $k_{i}^{0}$, with associated weights $w_{i}$. The regularization, if appropriately chosen, can help ensure that the optimized parameters remain physically sound while also preventing overfitting. The chosen weights $w_{g, m}$ for the energies were inversely proportional to their expected values $E_{g}^{0}$, and scaled so that a geometry with an associated $E_{g}^{0}=50 \mathrm{kcal} \mathrm{mol}^{-1}$ would have $w_{g}=1 \mathrm{kcal} \mathrm{mol}^{-1}$ (i.e., a penalty value of 25 if there was a $10 \%$ associated error). This ensured that relative errors, rather than absolute errors, were considered; such that configurations with higher associated energies and errors would not dominate the overall fitting. ${ }^{21}$ The associated weights $w_{g, m}$ for $F_{\text {int }}$ were all set to the unit value, since this gave them roughly the same weight in the fitting to $E_{\text {int }}$, given the magnitude of the final deviations (using LAMMPS 'real' units).

Different sets of constraints, $w_{i}$, for the LJ parameters and partial charges were tested; $10 \%$ variations on said parameters were assigned penalties of $10^{0}, 10^{1}, 10^{2}, 10^{3}$ or $10^{4}$, so that different Optimized FFs ranged from energy-dominated to parameter-dominated. Although starting guesses for the LJ parameters and partial charges were available from the Original FF, ${ }^{12}$ these had little physical motivation. Thus the selected Optimized FF assigned no penalties to these parameters, to obtain the global best fit. Thus, although the same opmitizations were carried out with different degrees of penalties, results are only 
reported here for the non-penalized results.

No information was available regarding the analytic shape of $\chi^{2}$ and parameter evaluations were relatively expensive to compute (initialising LAMMPS and retrieving the $E_{\text {int }}$ for the 30 configurations could take $\mathcal{O}(s)$ for each set of parameters). Therefore, we tested two different types of algorithm that require no knowledge of the Jacobian or Hessian: quasiNewtonian algorithms and particle swarm optimization (PSO).

Quasi-Newtonian algorithms are designed to find local minima close to an initial starting point. Several bound-constrained versions which are implemented in the SciPy Python package were tested; bound constrained limited-memory BFGS (L-BFGS-B), truncated Newton (TNC), trust-region reflective (TRF), and sequential least squares programming (SLSQP). We found L-BFGS-B and TNC to performed the best for our system when appropriately tuned and behave more robustly using different initial guesses. However, the necessity for initial estimates remained a drawback given the lack of physically motivated parameters for our system avaiable in the literature.

On the other hand, PSO algorithms are more suited to finding global minima by having a population of candidate solutions (particles) starting at different points in $k$-space where $\chi$ is evaluated. The position, $x_{i}$, and velocity, $v_{i}$, of each particle is determined at step $i$ according to the formula:

$$
\begin{aligned}
& \mathbf{v}_{i+1}=\omega \cdot \mathbf{v}_{i}+c_{1} \cdot \mathbf{r}_{1} \cdot\left(\mathbf{p}_{p}-\mathbf{x}_{i}\right)+c_{2} \cdot \mathbf{r}_{2} \cdot\left(\mathbf{p}_{g}-\mathbf{x}_{i}\right) \\
& \mathbf{x}_{i+1}=\mathbf{x}_{i}+\mathbf{v}_{i+1}
\end{aligned}
$$

where $\mathbf{p}_{p}$ is the best previous fit evaluated by the particle, $\mathbf{p}_{g}$ is the best global fit registered by any particle in the swarm, $\mathbf{r}_{1,2}$ are vectors of random numbers in the range $[0,1]$, and $\omega$, $c_{1}, c_{2}$ are weights for the different terms. Therefore, the particles feel a statistical attraction to their own and global recorded best positions, while the inertia weight determined by $w$ 
prevents the search space of the swarm from shrinking too fast. We chose $w=c_{1}=0.5$ and a lower weight $c_{2}=0.2$ to prevent such behaviour. We employed a dense swarm of 1100 particles for the safety of each optimization, which consistently converged after approximately 20 iterations.

Despite the stochastic nature of PSO, we found it consistently performed as well if not better than quasi-Newtonian algorithms. Furthermore, the algorithm was easily parallelized such that each particle was run on a different core. Thus, time efficiency, global fits (as opposed to local), and the non-requirement of initial guesses made PSO the preffered method for the optimization.

Of the 33 DFT configurations available, 29 were included in the training dataset, with the closest and most distant systems excluded to prevent them from skewing the overall fit. Regardless of the parameters chosen, the Optimized FF overestimated repulsion at very close distances to the surface, due to the unphysically steep repulsive term in the 12-6 LJ potential. ${ }^{22-24}$ A possible solution would be to fit interactions for all of the atom pairs with a Morse potential, but this is beyond the scope of this current study. Instead, 2 configurations with HAm molecules $<1.0 \AA$ from the surface were excluded from the optimization to prevent them from skewing the fitting. The configuration with the HAm molecule furthest from the surface $(3.6 \AA)$ had a DFT-calculated energy of only $0.01 \mathrm{kcal} \mathrm{mol}^{-1}$ (within the error of the DFT calculations), whereas our Optimized FFs predicted $\sim 0.1 \mathrm{kcal} \mathrm{mol}^{-1}$. Given that the fitting was minimized using relative errors, ${ }^{21}$ these geometries would dominate the overall fitting if they were included. Another relaxed geometry where the HAm relaxed with the $\mathrm{N}_{\text {amide }}$ close to the surface and the $\mathrm{O}_{\text {amide }}$ pointing away from the surface was also excluded from the force-matching. When this was included, the Optimized FF resulted in an unphysical $\mathrm{N}_{\text {amide }}-\mathrm{Fe}_{\text {surf }}$ distance $(0.2 \AA)$ in the large-scale NEMD simulations. Nevertheless, these 4 configurations are still included in Fig. 5 in the main text and the Optimized FF reproduces their $E_{\text {int }}$ values with remarkable accuracy. 


\section{Choice of parameters and potential models}

Initially, we attempted to parameterize an interface FF with the Lennard-Jones + Coulomb $(\mathrm{LJ}+\mathrm{C})$ potentials. Such a LJ+C FF would be desirable due to relatively high transferability to other similar systems using mixing rules.
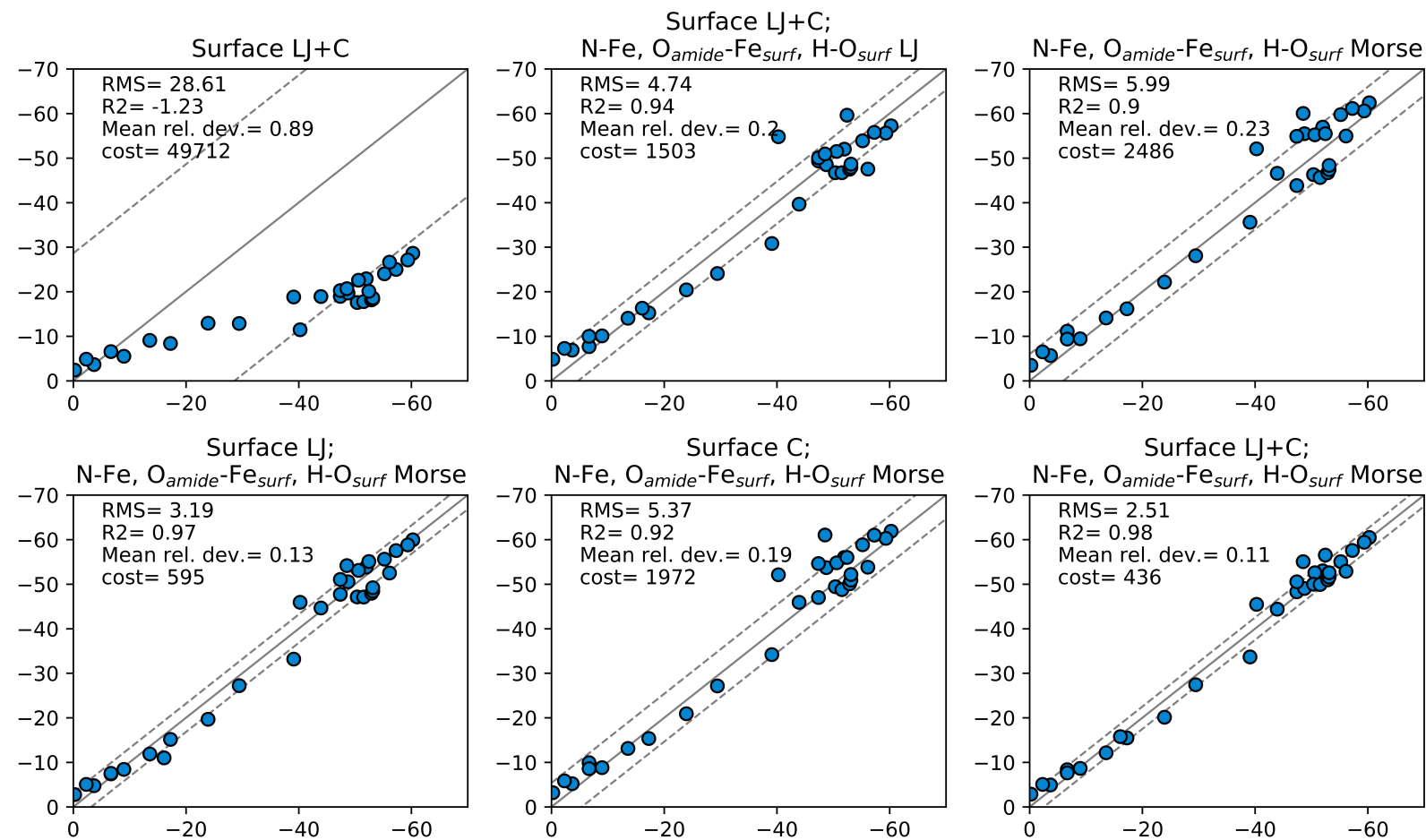

Figure S4: Comparison of the DFT vs. FF performance for different parameter optimizations. Parameters which were not optimized in each case were left as they were in the Original FF. ${ }^{12}$ Solid lines represent the ideal fitting, dashed lines represent RMS errors for each set optimization. Text in each plot includes the RMS deviation, the $R^{2}$ value of the fitting, the mean relative deviation and the optimized $\chi^{2}$ value (see eq. 1). The FF shown at the bottom right, with the most parameters fit, was the variant selected as the final Optimized $\mathrm{FF}$.

The first parameterization was performed only on the surface LJ+C parameters. We found that, even when parameters were completely unconstrained, no acceptable fitting could be achieved (see Fig. S4). While LJ is suitable to represent weak van der Waals interactions, it could not fit the strong interactions between the headgroup atoms and the surface without resulting in inaccurate repulsive and attractive $E_{\text {int }}$ at closer and further 
distances respectively. Moreover, the van der Waals interactions between the tailgroup and the surface atoms would not be well described by an optimization which was be dominated by the headgroup-surface interactions.

Hence, the next step was to explicitly parameterize the dominating headgroup-surface interactions. Charge transfer was apparent from the DFT calculations between the $\mathrm{O}_{\text {amide }}$ and the $\mathrm{Fe}_{\text {surf }}$ atoms, the $\mathrm{O}_{\text {amide }}$ and the $\mathrm{Fe}_{\text {surf }}$ atoms, as well as between the $\mathrm{H}_{\text {amide }}$ and $\mathrm{O}_{\text {surf }}$ atoms. We optimized different combinations of parameters and potential functions. While employing different LJ parameters all of the atom pairs yielded a clear improvement in the fitting, the energy-distance behaviour was not well reproduced (see Fig. S5). The use of Morse potentials, which include a parameter to describe the well width, ensured a better fittings across different distances. Given that the strong Morse interactions dominated the final $E_{\text {int }}$ compared to the weaker van der Waals and Coulombic interactions, different combinations of the latter were tested together with the Morse interactions to study their relative influence. 

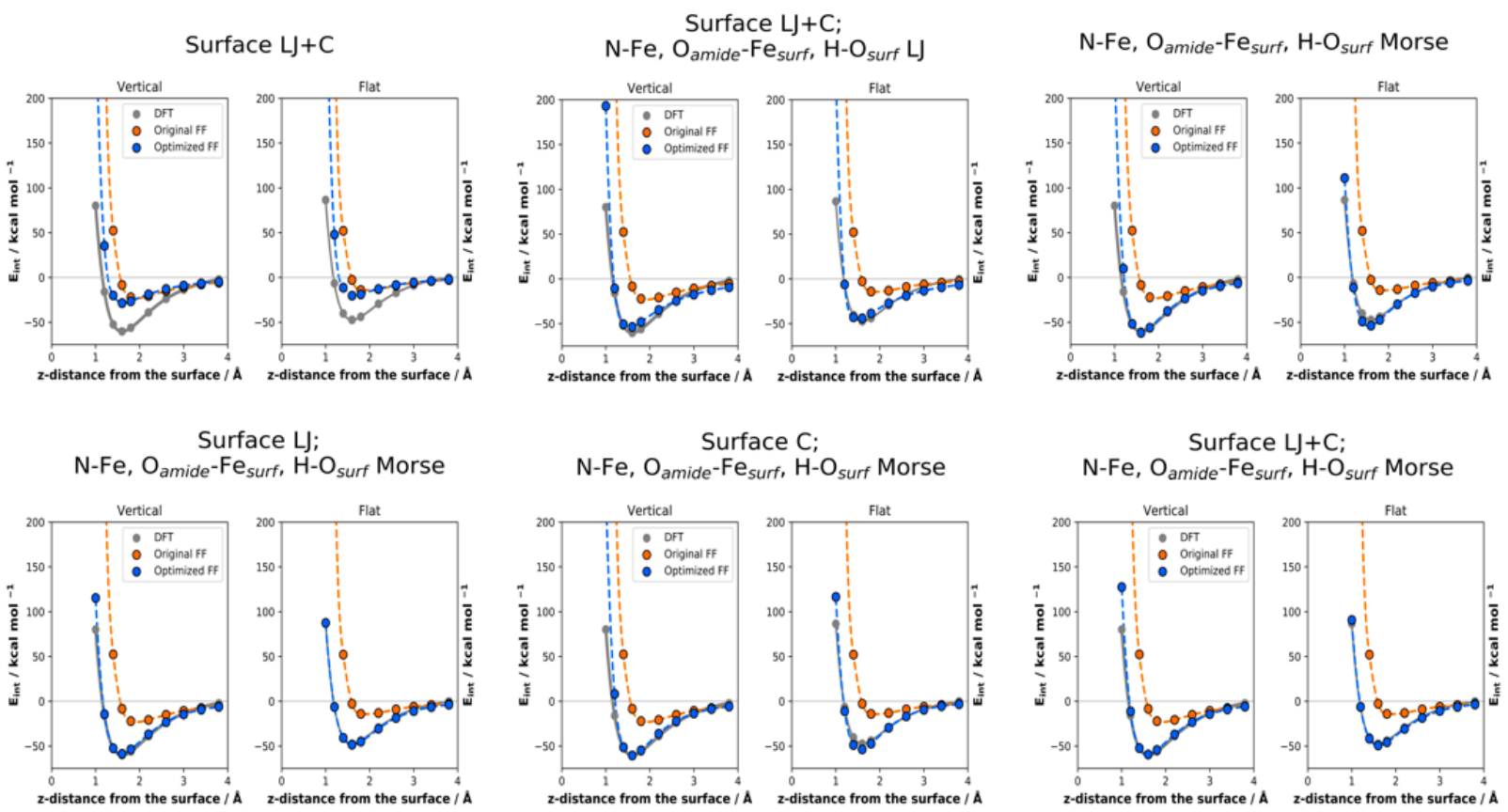

Figure S5: Comparison of $z$-scans of $E_{\text {int }}$ from DFT and different FF parameter sets (same as Fig.S4). The FF shown at the bottom right, with the most parameters fit, was the variant selected as the final Optimized FF.

Parameterizing only the Morse interactions, while leaving the surface $\mathrm{LJ}+\mathrm{C}$ from the Original $\mathrm{FF},{ }^{12}$ already yielded a good fit and much improved energy-distance behaviour over the $\mathrm{LJ}+\mathrm{C}$ variants. Parameterizing the surface $\mathrm{LJ}+\mathrm{C}$ parameters further improved the performance. Since the surface parameters in the Original FF had little physical motivation, we decided to alter these (values reported in the main text) to obtain the best possible match to the DFT calculations.

For the sake of completeness, we also include the parameters for a FF where the surface LJ+C are optimized collectively (top-left in Fig. S4-5), and for where the surface LJ+C interactions are parameterized alongside strong LJ interactions for the $\mathrm{O}_{\text {amide }}-\mathrm{Fe}_{\text {surf }}, \mathrm{N}_{\text {amide }}{ }^{-}$ $\mathrm{Fe}_{\text {surf }}$, and $\mathrm{H}_{\text {amide }}-\mathrm{O}_{\text {surf }}$ atom pairs (top-middle in Fig. S4-5). Note that, despite the reasonable good agreement with the DFT $E_{a d s}$ values for the latter, the required parameters are physically unjustifiable. Most notably, the strength of the $\mathrm{O}_{\text {amide }}-\mathrm{Fe}_{\text {surf }}$ interaction is virtually zero, while the $\mathrm{N}_{\text {amide }}-\mathrm{Fe}_{\text {surf }}$ has a well-depth of $5.77 \mathrm{kcal} \mathrm{mol}^{-1}$, which is an order 
of magnitude stronger than the types of interaction which LJ potentials usually represent.

Table S2: Parameters for the two LJ-based optimised FF tested

\begin{tabular}{lccc}
\hline & $\epsilon / \mathrm{kcal} \mathrm{mol}^{-1}$ & $\sigma / \AA$ & $q / \mathrm{e}$ \\
Only surface $L J+C$ & 0.1 & 2.0 & 0.9 \\
$\mathrm{Fe}_{\text {surf }}$ & 0.4 & 2.2 & -0.6 \\
$\mathrm{O}_{\text {surf }}$ & & & \\
\hline & $\epsilon / \mathrm{kcal} \mathrm{mol}^{-1}$ & $\sigma / \AA$ & $q / \mathrm{e}$ \\
Surface LJ+C, & & & \\
Strong interactions LJ & & & \\
$\mathrm{Fe}_{\text {surf }}$ & 0.44 & 2.29 & 0.51 \\
$\mathrm{O}_{\text {surf }}$ & 0.24 & 2.47 & -0.34 \\
$\mathrm{O}_{\text {amide }}-\mathrm{Fe}_{\text {surf }}$ & 0.0002 & 3.08 & - \\
$\mathrm{N}_{\text {amide }}-\mathrm{Fe}_{\text {surf }}$ & 5.77 & 2.97 & - \\
$\mathrm{H}_{\text {amide }}-\mathrm{O}_{\text {surf }}$ & 1.64 & 1.64 & - \\
\hline
\end{tabular}

* Parameters for HAm remain unmodified from L-OPLS-AA, ${ }^{25-27}$ and the remaining LJ parameters are determined by geometric mean mixing rules.

\section{Comparing force-fields for $n$-hexane adsorption}

The Optimized FF was also tested for the interaction between a model lubricant ( $n$-hexane) molecule and the $\alpha-\mathrm{Fe}_{2} \mathrm{O}_{3}(0001)$ surface. Although the Optimized FF was not parameterized for this system, it is envisaged that it will frequently be used in the presence of $n$-alkanes, ${ }^{16}$ so the performance for such systems should be at least equivalent to the available alternatives. ${ }^{11-13}$ The most stable adsorption structure, with $n$-hexane in a flat conformation on the $\alpha-\mathrm{Fe}_{2} \mathrm{O}_{3}(0001)$ surface, is shown in Fig. S2. This geometry was used for single-point calculations where $n$-hexane was moved closer to, or further from, the surface ( $z$-scan). The DFT $E_{\text {int }}$ values were compared to results obtained from the Optimized FF, ClayFF, ${ }^{11}$ as well as the FFs due to Berro et al. ${ }^{12}$ and Savio et al., ${ }^{13}$ as shown in Fig. S6. 


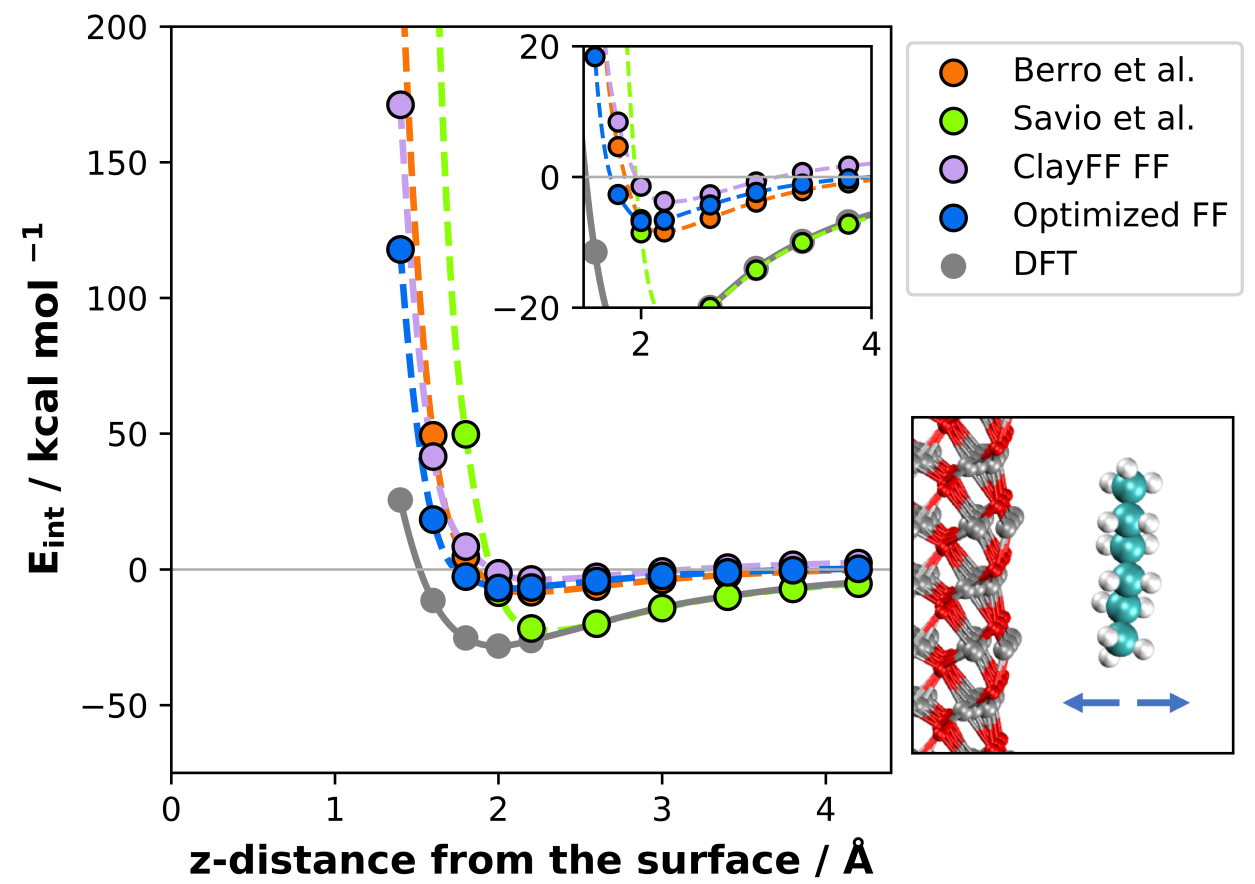

Figure S6: $z$-Scans of $E_{\text {int }}$ for isolated $n$-hexane molecule in flat conformation on $\alpha$ $\mathrm{Fe}_{2} \mathrm{O}_{3}(0001)$ from Berro at al., ${ }^{12}$ Savio et al., ${ }^{13}$ Optimized FF, and DFT calculations. Lines are spline interpolations to the data.

Compared to DFT, $E_{a d s}$ for $n$-hexane was significantly underestimated by the Optimized $\mathrm{FF}$, the Original $\mathrm{FF}^{12}$ and particularly ClayFF on $\alpha$ - $\mathrm{Fe}_{2} \mathrm{O}_{3}(0001) .{ }^{11}$ Since $n$-hexane is relatively nonpolar, it is unsurprising that this is the case for ClayFF, ${ }^{11}$ which was designed for the interactions between metal oxides and water molecules which are dominated by electrostatics. The FF due to Savio et al. ${ }^{13}$ was more accurate $\left(E_{a d s} \sim 70 \%\right.$ of DFT) in this respect; however, this $\mathrm{FF}$ overestimates $z_{e q}$ and short-ranged repulsive energies far more than the other FFs. The Optimized FF and ClayFF give better with DFT in terms of $z_{e q}$ and short-ranged repulsion. It can be concluded that, while the Optimized FF is far from perfect for $n$-hexane adsorption on $\alpha-\mathrm{Fe}_{2} \mathrm{O}_{3}(0001)$, as this was not the objective of the parameterization, it does not significantly hinder the performance when compared to other FFs in the literature. Improved performance for $n$-alkane adsorption would require Morse parameters be developed, which is beyond the scope of this study. 


\section{Additional NEMD results}

The following figures show the, number density profiles, atomic mass density profiles, and velocity profiles from the sliding phase of the NEMD simulations. These include the results for the top wall moving in the $-x,+y$ and $-y$, i.e. those directions not included in the main text $(+x)$. The $z$-dimension was partitioned into $0.05 \AA$ Abins to resolve the atomic $z$-coordinates for the number density and mass density profiles and 1.0 Åfor the velocity profiles. Block-averaged values are taken every 20 ps after 0.5 ns i.e. after systems reach a nonequilibrium steady state.

\section{Number density profiles}
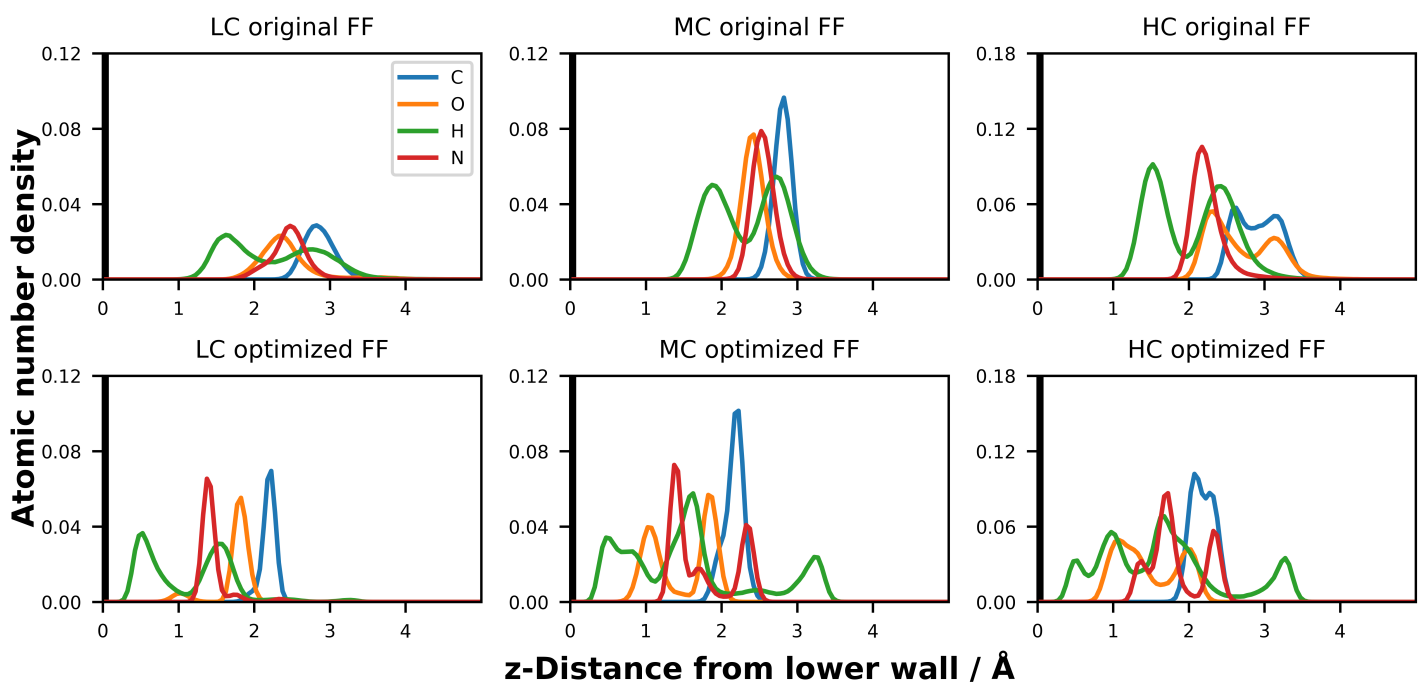

Figure S7: Atomic number density profiles, $-x$ direction. 

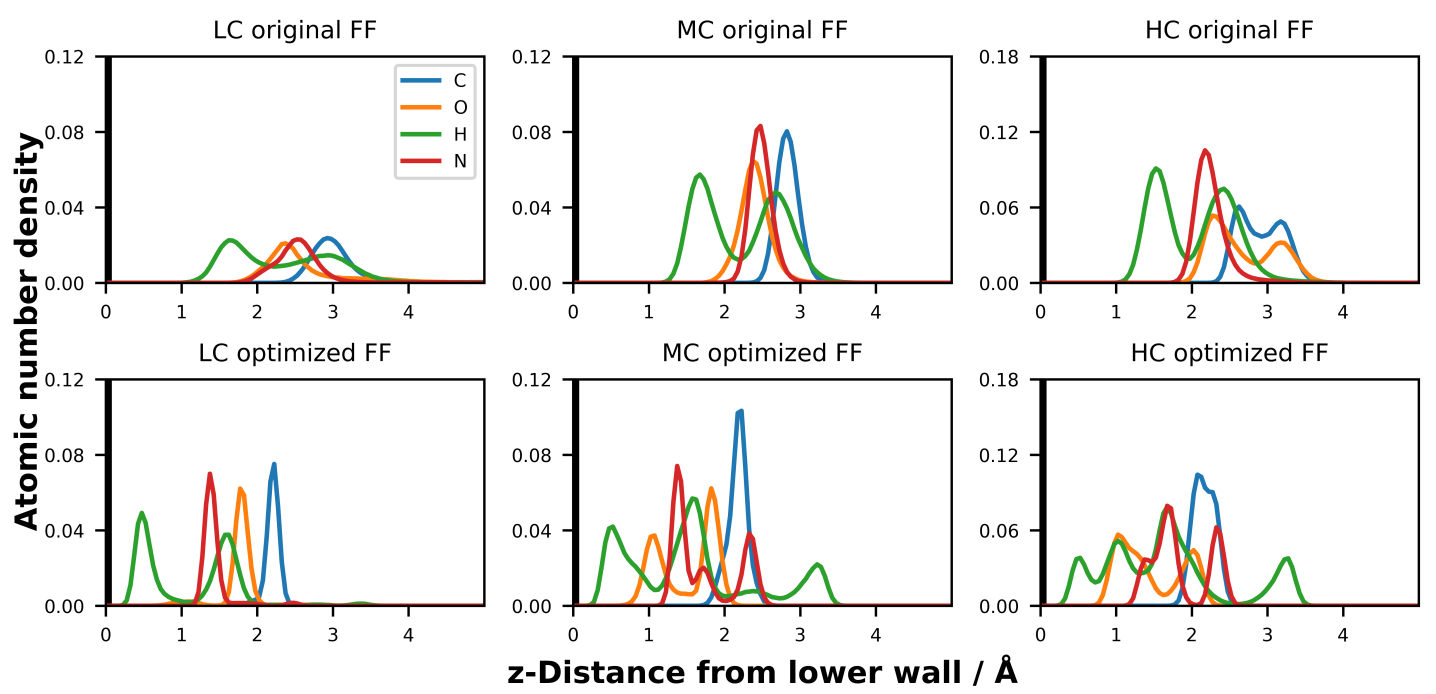

Figure S8: Atomic number density profiles, $y$ direction.
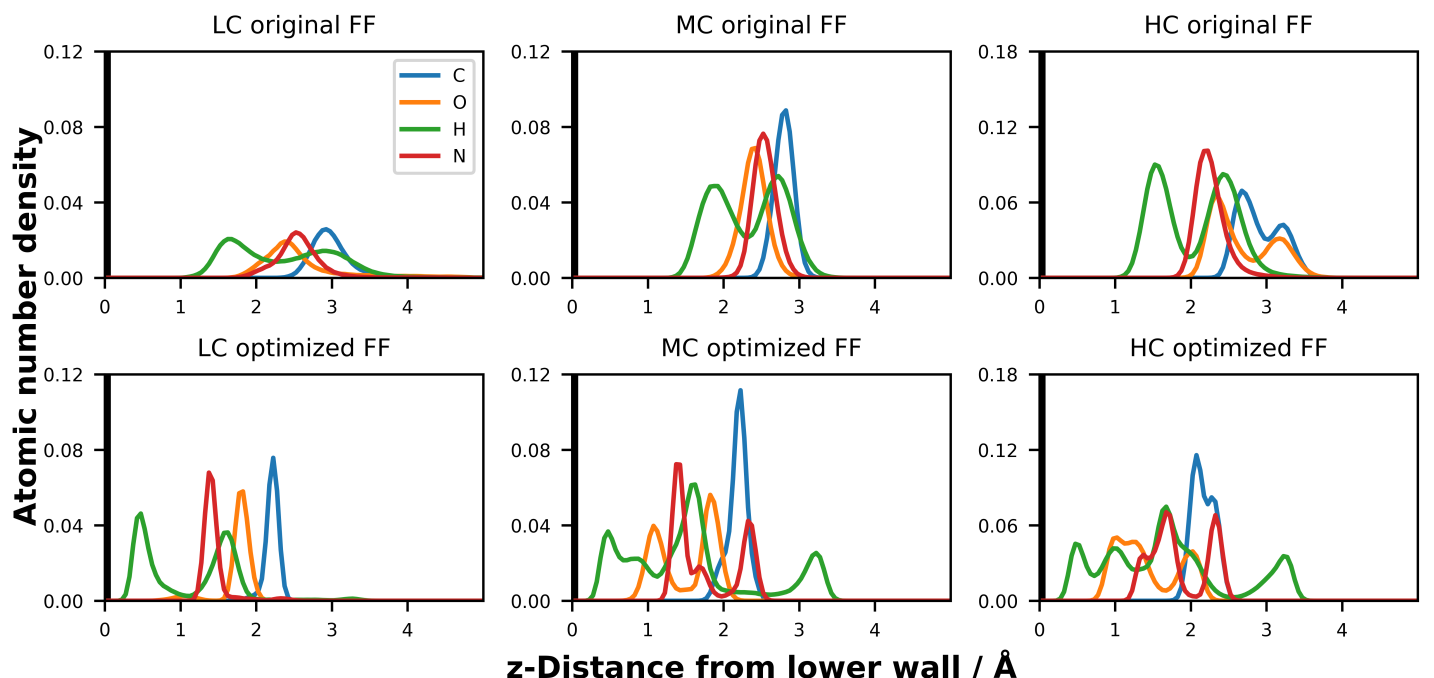

Figure S9: Atomic number density profiles, $-y$ direction.

\section{Atomic Mass Density and Velocity Profiles}

Velocity profiles for atomic velocities along the sliding direction in dashed purple lines, overlaid on the corresponding mass density profiles. 


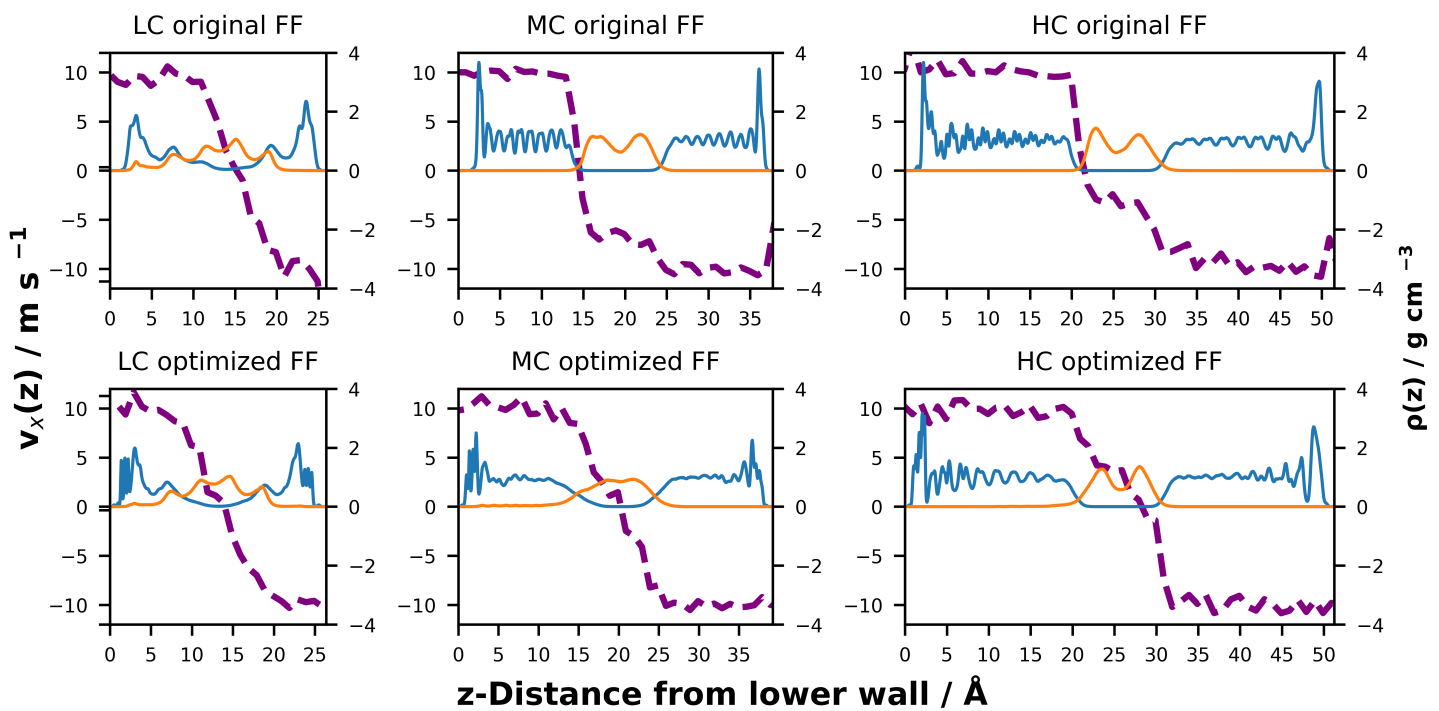

Figure S10: Velocity profiles, $-x$ direction.
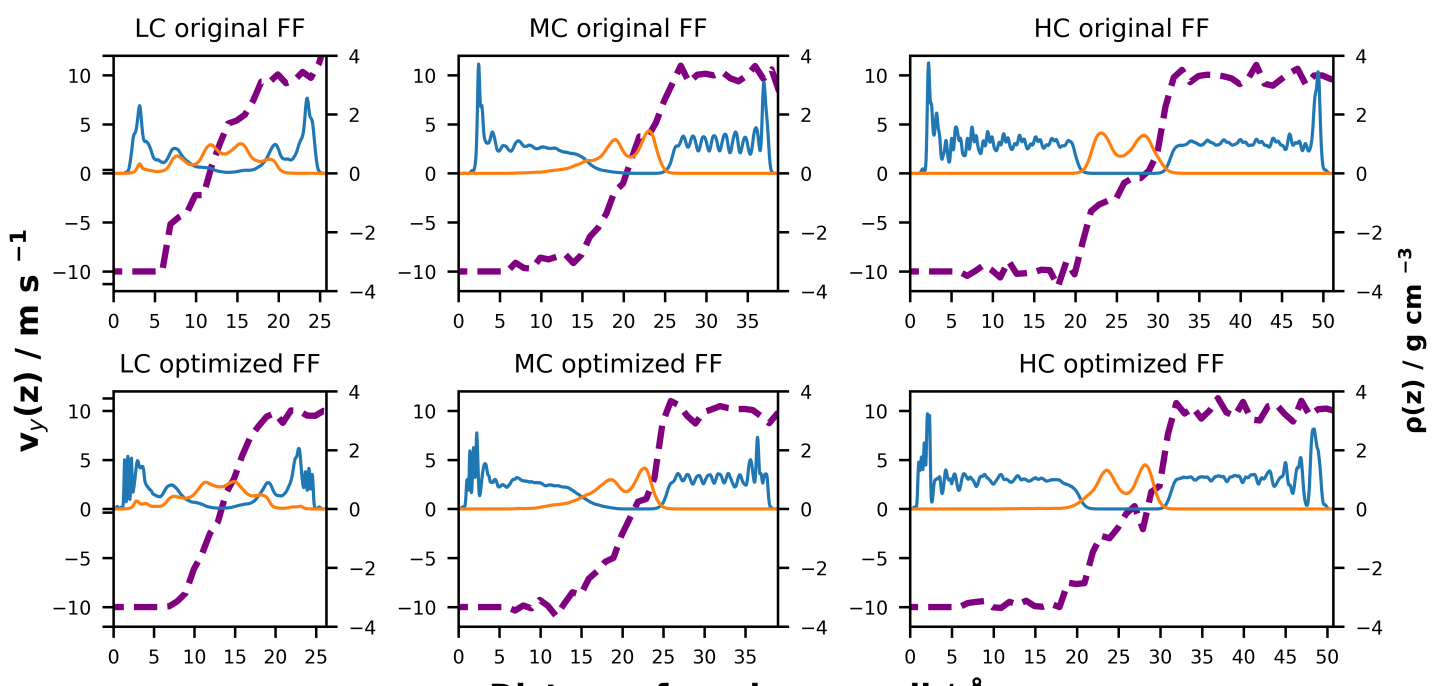

z-Distance from lower wall / $\AA$

Figure S11: Velocity profiles, $y$ direction. 


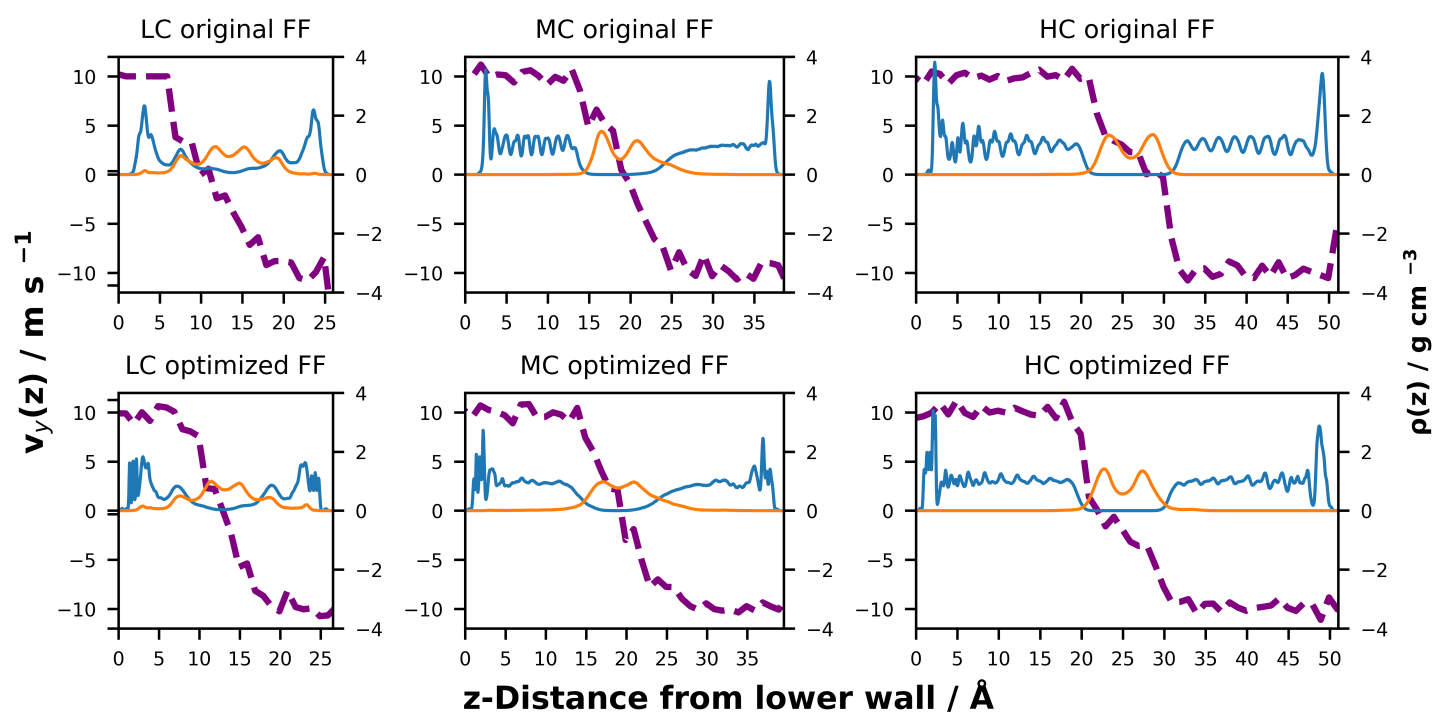

Figure S12: Velocity profiles, $-y$ direction.

\section{References}

(1) Gattinoni, C.; Ewen, J. P.; Dini, D. Adsorption of Surfactants on $\alpha$-Fe2O3(0001): A Density Functional Theory Study. J. Phys. Chem. C 2018, 122, 20817-20826.

(2) Gao, D. Z.; Federici Canova, F.; Watkins, M. B.; Shluger, A. L. Efficient parametrization of complex molecule-surface force fields. J. Comput. Chem. 2015, 36, 1187-1195.

(3) Huan, T. D.; Batra, R.; Chapman, J.; Krishnan, S.; Chen, L.; Ramprasad, R. A universal strategy for the creation of machine learning-based atomistic force fields. npj Comput. Mater. 2017, 3, 37.

(4) Tromp, S.; Joly, L.; Cobian, M.; Fillot, N. Chemical Physics at Interfaces within a Refrigerant-Lubricated Contact: From Electronic Structure to Large-Scale Molecular Dynamics Simulations. J. Phys. Chem. C 2018, 122, 5420-5429.

(5) Raabe, G.; Maginn, E. J. Molecular Modeling of the Vapor-Liquid Equilibrium Prop- 
erties of the Alternative Refrigerant 2,3,3,3-Tetrafluoro-1-propene (HFO-1234yf). J. Phys. Chem. Lett. 2010, 1, 93-96.

(6) Ta, T. D.; Tieu, A. K.; Zhu, H.; Kosasih, B. Adsorption of Normal-Alkanes on Fe(110), $\mathrm{FeO}(110)$, and $\mathrm{Fe} 2 \mathrm{O} 3(0001)$ : Influence of Iron Oxide Surfaces. J. Phys. Chem. C 2015, 119, 12999-13010.

(7) Sun, H. COMPASS: An ab initio force-field optimized for condensed-phase applications - Overview with details on alkane and benzene compounds. J. Phys. Chem. B 1998, 102, 7338-7364.

(8) Sun, H.; Jin, Z.; Yang, C.; Akkermans, R. L.; Robertson, S. H.; Spenley, N. A.; Miller, S.; Todd, S. M. COMPASS II: extended coverage for polymer and drug-like molecule databases. J. Mol. Model. 2016, 22, 1-10.

(9) Huang, X.; Ramadugu, S. K.; Mason, S. E. Surface-Specific DFT + U Approach Applied to $\alpha$-Fe2O3(0001). J. Phys. Chem. C 2016, 120, 4919-4930.

(10) Heinz, H.; Lin, T. J.; Kishore Mishra, R.; Emami, F. S. Thermodynamically consistent force fields for the assembly of inorganic, organic, and biological nanostructures: The INTERFACE force field. Langmuir 2013, 29, 1754-1765.

(11) Cygan, R. T.; Liang, J. J.; Kalinichev, A. G. Molecular models of hydroxide, oxyhydroxide, and clay phases and the development of a general force field. J. Phys. Chem. B 2004, 108, 1255-1266.

(12) Berro, H.; Fillot, N.; Vergne, P. Molecular dynamics simulation of surface energy and ZDDP effects on friction in nano-scale lubricated contacts. Tribol. Int. 2010, 43, 18111822.

(13) Savio, D.; Fillot, N.; Vergne, P.; Zaccheddu, M. A Model for Wall Slip Prediction of 
Confined n-Alkanes: Effect of Wall-Fluid Interaction Versus Fluid Resistance. Tribol. Lett. 2012, 46, 11-22.

(14) Doig, M.; Warrens, C. P.; Camp, P. J. Structure and friction of stearic acid and oleic acid films adsorbed on iron oxide surfaces in squalane. Langmuir 2014, 30, 186-195.

(15) Doig, M.; Camp, P. J. The structures of hexadecylamine films adsorbed on iron-oxide surfaces in dodecane and hexadecane. Phys. Chem. Chem. Phys. 2015, 17, 5248-5255.

(16) Ewen, J. P.; Gattinoni, C.; Morgan, N.; Spikes, H. A.; Dini, D. Nonequilibrium Molecular Dynamics Simulations of Organic Friction Modifiers Adsorbed on Iron Oxide Surfaces. Langmuir 2016, 32, 4450.

(17) Ewen, J. P.; Gattinoni, C.; Thakkar, F. M.; Morgan, N.; Spikes, H.; Dini, D. A Comparison of Classical Force-Fields for Molecular Dynamics Simulations of Lubricants. Materials. 2016, 9, 651.

(18) Ewen, J. P.; Kannam, S. K.; Todd, B. D.; Dini, D. Slip of alkanes confined between surfactant monolayers adsorbed on solid surfaces. Langmuir 2018, 34, 3864-3873.

(19) Jaishankar, A.; Jusufi, A.; Vreeland, J. L.; Deighton, P.; Pellettiere, J. R.; Schilowitz, A. M. Adsorption of stearic acid at the iron oxide/oil interface - theory, experiments and modeling. Langmuir 2019,

(20) Jorgensen, W. L.; Madura, J. D.; Swenson, C. J. Optimized intermolecular potential functions for liquid hydrocarbons. J. Am. Chem. Soc. 1984, 106, 6638-6646.

(21) Herbers, C. R.; Li, C.; van der Vegt, N. F. A. Grand Challenges in Quantum-Classical Modeling of Molecule- Surface Interactions. J. Comput. Chem. 2013, 34, 1177-1188.

(22) Kong, L. T.; Denniston, C.; Müser, M. H.; Qi, Y. Non-bonded force field for the interaction between metals and organic molecules: a case study of olefins on aluminum. Phys. Chem. Chem. Phys. 2009, 11, 10195-10203. 
(23) Johnston, K.; Herbers, C. R.; Van Der Vegt, N. F. Development of classical moleculesurface interaction potentials based on density functional theory calculations: Investigation of force field representability. J. Phys. Chem. C 2012, 116, 19781-19788.

(24) O'Connor, T. C.; Andzelm, J.; Robbins, M. O. AIREBO-M: A reactive model for hydrocarbons at extreme pressures. J. Chem. Phys. 2015, 142, 024903.

(25) Jorgensen, W. L.; Maxwell, D. S.; Tirado-Rives, J. Development and testing of the OPLS all-atom force field on conformational energetics and properties of organic liquids. J. Am. Chem. Soc. 1996, 118, 11225-11236.

(26) Price, M. L. P.; Ostrovsky, D.; Jorgensen, W. L. Gas-phase and liquid-state properties of esters, nitriles, and nitro compounds with the OPLS-AA force field. J. Comput. Chem. 2001, 22, 1340-1352.

(27) Siu, S. W. I.; Pluhackova, K.; Bockmann, R. A. Optimization of the OPLS-AA Force Field for Long Hydrocarbons. J. Chem. Theory Comput. 2012, 8, 1459-1470. 
